\title{
Synthesis of Novel Enantiopure Fluorinated Building Blocks from Acyclic Chiral
}

\author{
Allylsilanes \\ Matthew Tredwell, Kenny Tenza, Mª Carmen Pacheco, Véronique Gouverneur* \\ University of Oxford, Chemistry Research Laboratory, 12 Mansfield Road, OX1 3TA \\ $\operatorname{Oxford}(U K)$
}

\section{Supporting Information}

\section{General Information}

${ }^{1} \mathrm{H}$ NMR spectra were recorded in deuterated solvents using Bruker DPX200, DPX400, AV400 and AVC500 spectrometers, calibrated using residual undeuterated solvent as an internal reference. ${ }^{13} \mathrm{C}$ NMR spectra were recorded in deuterated solvents using Bruker DPX200, DPX400, AV400 and AVC500 spectrometers. ${ }^{19} \mathrm{~F}$ spectra were recorded on a AV400 spectrometer. Chemical shifts $(\delta)$ are quoted in parts per million (ppm) and coupling constants $(J)$ are measured in hertz $(\mathrm{Hz})$. The following abbreviations are used to describe multiplicities s=singlet, d=doublet, $\mathrm{t}=$ triplet, $\mathrm{q}=$ quartet, $\mathrm{b}=$ broad, $\mathrm{m}=$ multiplet. NMR were processed in either MestRe-C or $\mathrm{ACD} /$ SpecManager. IUPAC names were obtained using the ACD/I-lab service. Mass spectra were recorded on Micromass GCT (CI), Autospec-oaTof instruments. Optical rotations were determined on a Perkin Elmer 241 polarimeter in a $1 \mathrm{dm}$ cell.

$[\alpha]_{\mathrm{D}}$ values are given in $10^{-1} \mathrm{deg} \mathrm{cm}^{2} \mathrm{~g}^{-1}$. IR spectra were recorded as thin films on $\mathrm{NaCl}$ plates in solution in $\mathrm{CHCl}_{3}$ on a Bruker Tensor 27 FT-IR spectrometer. Absorptions are measured in wavenumbers and only peaks of interest are reported. 
All reactions requiring anhydrous conditions were conducted in dried apparatus under an inert atmosphere of argon or nitrogen. Solvents were dried and purified before use according to standard procedures. All reactions were monitored by TLC using Merck Kiesegel $60 \mathrm{~F}_{254}$ plates. Visualisation of the reaction components was achieved using U.V fluorescence $(254 \mathrm{~nm})$ and $\mathrm{KMnO}_{4}$ stain. Column chromatography was carried out over Merck silica gel C60 $(40-60 \mu \mathrm{m})$.

\section{(4R)-3-((E)-2-butenoyl)-4-(phenylmethyl)-2-oxazolidinone (3)}

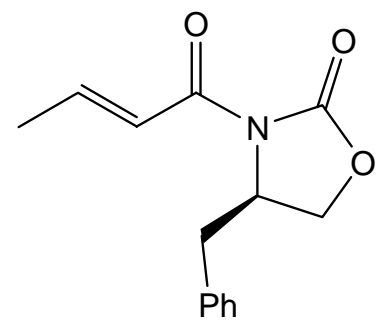

To a stirred solution of (R)-4-benzyl-2-oxazolidinone (5 g, $28.2 \mathrm{mmol})$ in THF (94 $\mathrm{mL})$ at $-78{ }^{\circ} \mathrm{C}$ was added 1 eq of $\mathrm{n}-\mathrm{BuLi}(2.3 \mathrm{M}, 12.26 \mathrm{~mL})$. After $15 \mathrm{~min} 1.1 \mathrm{eq}$ of crotonyl chloride (31 mmol, $2.97 \mathrm{~mL}$ ) was added directly to the solution. The solution was allowed to stir for $30 \mathrm{~min}$ at $-78{ }^{\circ} \mathrm{C}$, then $15 \mathrm{~min}$ at $0{ }^{\circ} \mathrm{C}$. The reaction was quenched with saturated $\mathrm{NH}_{4} \mathrm{Cl}$ solution then extracted with ether. The solvents were removed in vacuo and the crude product recrystallised from hexane: ethylacetate to yield a white solid $(10.6 \mathrm{~g}, 77 \%) ;{ }^{1} \mathrm{H}$ NMR $\left(400 \mathrm{MHz}, \mathrm{CDCl}_{3}\right)$ : $\delta=7.18-7.36(\mathrm{~m}$, 7H), $4.74(\mathrm{~m}, 1 \mathrm{H}), 4.1-4.14(\mathrm{~m}, 2 \mathrm{H}), 3.34(\mathrm{dd}, J=3.3,13.4,1 \mathrm{H}), 2.80(\mathrm{dd}, J=9.6$, $13.4,1 \mathrm{H}), 1.99(\mathrm{~d}, J=5.3) ;{ }^{13} \mathrm{C} \mathrm{NMR}\left(100 \mathrm{MHz}, \mathrm{CDCl}_{3}\right): \delta=164.9,153.4,146.9$, 135.4, 129.4, 128.9, 127.3, 121.9, 66.1, 55.3, 37.9, 18.5; IR $\left(\mathrm{CHCl}_{3}\right): v 1780 \mathrm{~cm}^{-1}$; MS $\left(\mathrm{CI}\left(\mathrm{NH}_{3}\right)\right): m / z 246\left(\mathrm{M}+\mathrm{H}^{+}\right) ;[\alpha]_{\mathrm{D}}^{23}=-70.6^{\circ}\left(c\right.$ 0.5, $\left.\mathrm{CHCl}_{3}\right)$. Known compound: Evans, D. A.; Chapman, K. T.; Bisaha, J. J. Am. Chem. Soc. 1984, 106, 4261-4263. 
(4R)-4-benzyl-3-[(2R)-2-benzylbut-3-enoyl]-1,3-oxazolidin-2-one (4)

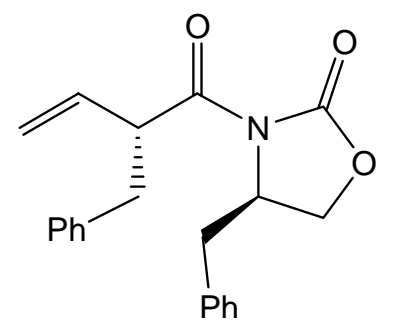

To a stirred solution of diisopropylamine $(20.46 \mathrm{mmol}, 2.85 \mathrm{~mL})$ in THF $(73 \mathrm{~mL})$ at $78{ }^{\circ} \mathrm{C}$ was added n-BuLi (2.3 M, 8.89 mL). After 10 minutes HMPA (20.46 mmol, 3.5 $\mathrm{mL}$ ) was added and the mixture stirred for 30 minutes at $-78^{\circ} \mathrm{C}$. Compound $\mathbf{3}$ (18.6 mmol, $4.56 \mathrm{~g})$ in THF (36 mL) was added at $-78{ }^{\circ} \mathrm{C}$ followed 15 minutes later by benzyl bromide $(55.8 \mathrm{mmol}, 6.6 \mathrm{~mL})$. After 20 minutes the reaction was allowed to warm to $-10{ }^{\circ} \mathrm{C}$ and stirred at this temperature for 90 minutes. Dilute $\mathrm{HCl}$ was added and the aqueous layer extracted with ether. The combined organic phases were washed with saturated $\mathrm{NaCl}$ solution, dried $\left(\mathrm{MgSO}_{4}\right)$, filtered and the solvent removed in vacuo to yield an orange oil. The crude product was purified by column chromatography to yield a white solid $(3.5 \mathrm{~g}, 56 \%) ;{ }^{1} \mathrm{H}$ NMR $\left(400 \mathrm{MHz}, \mathrm{CDCl}_{3}\right): \delta=$ 7.0-7.34 (m, 10H), 5.91-6.1 (m, 1H), 5.15-5.25 (m, 2H), $4.92(\mathrm{q}, 1 \mathrm{H}), 4.65(\mathrm{~m}, 1 \mathrm{H})$, 4.11-4.17 (m, 1H), 4.06-4.10 (dd, $J=2.7,9.1,1 \mathrm{H}), 3.27(\mathrm{dd}, J=8.6,13.4,1 \mathrm{H}), 3.02$ $(\mathrm{dd}, J=3.3,13.4), 2.92(\mathrm{dd}, J=6.8,13.6,1 \mathrm{H}), 2.57(\mathrm{dd}, J=9.1,13.6,1 \mathrm{H}) ;{ }^{13} \mathrm{C} \mathrm{NMR}$ $\left(100 \mathrm{MHz}, \mathrm{CDCl}_{3}\right): \delta=173.4,152.9,138.6,135.4,135.0,129.5,129.4,128.9,128.3$, 127.3, 126.5, 118.4, 65.8, 55.1, 49.1, 38.6, 37.6; IR $\left(\mathrm{CHCl}_{3}\right): v$ 3424, 2255, 1779, 1696, 1636, 1497, 1455, 1386, 1212, $1104 \mathrm{~cm}^{-1} ; \mathrm{MS}\left(\mathrm{CI}\left(\mathrm{NH}_{3}\right)\right): \mathrm{m} / z 336\left(\mathrm{M}+\mathrm{H}^{+}\right)$; HMRS required for $\mathrm{C}_{21} \mathrm{H}_{22} \mathrm{NO}_{3}\left([\mathrm{M}]^{+}\right) 336.1604$ found 336.1599; $[\alpha]_{\mathrm{D}}^{23}=-67.8^{\mathrm{o}}(c$, $\left.1 \mathrm{CHCl}_{3}\right) ; \mathrm{Mp} 86-88{ }^{\circ} \mathrm{C}$. 


\section{Single-crystal X-ray diffraction report for (4R)-4-benzyl-3-[(2R)- 2-benzylbut-3-enoyl]-1,3-oxazolidin-2-one, $\mathrm{C}_{21} \mathrm{H}_{21} \mathrm{NO}_{3}$}

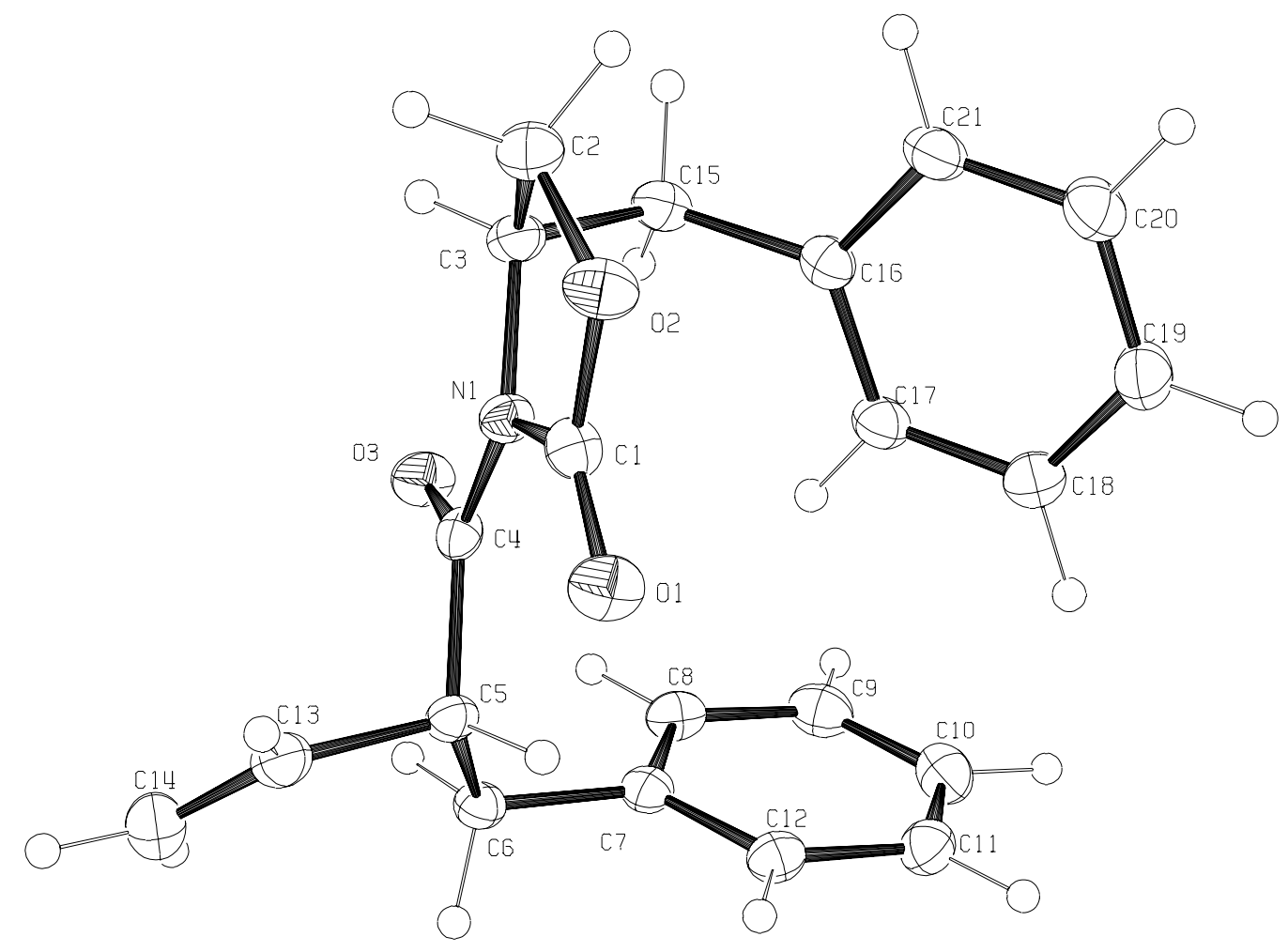

Crystals of $(4 R)$-4-benzyl-3-[(2R)-2-benzylbut-3-enoyl]-1,3-oxazolidin-2one were grown by recrystallisation from ethyl acetate. A large single crystal was cut to give a fragment having dimensions approximately $0.30 \times 0.36 \times$ $0.36 \mathrm{~mm}$. This was mounted on a glass fibre using perfluoropolyether oil and cooled rapidly to $150 \mathrm{~K}$ in a stream of cold $\mathrm{N}_{2}$ using an Oxford Cryosystems CRYOSTREAM unit. Diffraction data were measured using an Enraf-Nonius KappaCCD diffractometer (graphite-monochromated $\mathrm{MoK}_{\alpha}$ radiation, $\lambda=$ $0.71073 \AA$ ). Intensity data were processed using the DENZO-SMN package ${ }^{1}$.

Examination of the systematic absences of the intensity data showed the space group to be either $P 2_{1}$ or $P 2_{1} / \mathrm{m}$. The structure was solved in the space group $P 2_{1}$ using the direct-methods program SIR92 ${ }^{2}$, which located all non-hydrogen atoms. Subsequent full-matrix least-squares refinement was carried out using the CRYSTALS program suite ${ }^{3}$. Coordinates and anisotropic thermal parameters of all non-hydrogen atoms were refined. Hydrogen atoms were positioned geometrically after each cycle of refinement. A 3-term Chebychev polynomial weighting scheme was applied. Refinement converged satisfactorily to give $R$ $=0.0305, \mathrm{wR}=0.0303$.

Attached is a thermal ellipsoid plot (ORTEP- $3^{4}$ ) at $40 \%$ probability. A summary of crystallographic data is given below, as are full lists of atomic 
coordinates, anisotropic thermal parameters and those bond lengths and angles not concerning $\mathrm{H}$ atoms.

\section{References:}

1 Z. Otwinowski and W. Minor, Processing of X-ray Diffraction Data Collected in Oscillation Mode, Methods Enzymol., 1997, 276, Eds C. W. Carter and R. M. Sweet, Academic Press.

2 A. Altomare, G. Cascarano, G. Giacovazzo, A. Guagliardi ,M. C. Burla, G. Polidori and M. Camalli, J. Appl. Cryst. 1994, 27, 435.

3 CRYSTALS Issue 12, P. W. Betteridge, J. R. Cooper, R. I. Cooper, K. Prout and D. J. Watkin, J. Appl. Cryst., 2003, 36, 1487

$4 \quad$ ORTEP-3 v. 1.0.2, C. K. Johnson and M. K. Burnett, 1998.

Table 1: Crystal data and refinement details

\begin{tabular}{|c|c|}
\hline Crystal identification & ARC851 \\
\hline Chemical formula & $\mathrm{C}_{21} \mathrm{H}_{21} \mathrm{NO}_{3}$ \\
\hline Formula weight & 335.40 \\
\hline Temperature $(\mathrm{K})$ & 150 \\
\hline Wavelength $(\AA)$ & 0.71073 \\
\hline Crystal system & Monoclinic \\
\hline Space group & $P 2_{1}$ \\
\hline$a(\AA)$ & $9.5701(3)$ \\
\hline$b(\AA)$ & $10.0377(3)$ \\
\hline$c(\AA)$ & $9.7704(3)$ \\
\hline$\alpha\left(^{\circ}\right)$ & 90 \\
\hline$\beta\left(^{\circ}\right)$ & 111.8904(15) \\
\hline$\gamma\left({ }^{\circ}\right)$ & 90 \\
\hline Cell volume $\left(\AA^{3}\right)$ & $870.89(5)$ \\
\hline Z & 2 \\
\hline Calculated density $\left(\mathrm{Mg} / \mathrm{m}^{3}\right)$ & 1.279 \\
\hline Absorption coefficient $\left(\mathrm{mm}^{-1}\right)$ & 0.085 \\
\hline$F_{000}$ & 356 \\
\hline Crystal size $(\mathrm{mm})$ & $0.30 \times 0.36 \times 0.36$ \\
\hline Description of crystal & Colourless fragment \\
\hline Absorption correction & Semi-empirical from equivalent reflections \\
\hline Transmission coefficients (min,max) & $0.96,0.97$ \\
\hline$\theta$ range for data collection $\left({ }^{\circ}\right)$ & $5.0 \leq \theta \leq 27.5$ \\
\hline Index ranges & $-12 \leq h \leq 11,0 \leq k \leq 12,0 \leq I \leq 12$ \\
\hline Reflections measured & 8985 \\
\hline Unique reflections & 2078 \\
\hline $\mathrm{R}_{\text {int }}$ & 0.050 \\
\hline Observed reflections $(\mathrm{I}>3 \sigma(\mathrm{I}))$ & 1540 \\
\hline Refinement method & Full-matrix least-squares on $F$ \\
\hline Parameters refined & 226 \\
\hline Weighting scheme & Chebychev 3-term polynomial \\
\hline Goodness of fit & 1.1786 \\
\hline $\mathrm{R}$ & 0.0305 \\
\hline $\mathrm{wR}$ & 0.0303 \\
\hline
\end{tabular}


\begin{tabular}{|l|l|}
\hline Residual electron density $(\min , \max )\left(\mathrm{e}^{-3}\right)$ & $-0.16,0.14$ \\
\hline
\end{tabular}

Table 2: Atomic coordinates and equivalent isotropic thermal parameters $\left(\AA^{2}\right)$ of non-hydrogen atoms

\begin{tabular}{|c|c|c|c|c|}
\hline Atom & $\mathbf{X}$ & $\mathrm{y}$ & $\mathrm{z}$ & $\mathrm{U}_{\text {equiv }}$ \\
\hline & & & & \\
\hline $\mathrm{N}(1)$ & $0.5020(2)$ & $0.48462(19)$ & $0.2404(2)$ & 0.0228 \\
\hline $\mathrm{C}(1)$ & $0.5389(3)$ & $0.3644(2)$ & $0.1924(2)$ & 0.0261 \\
\hline $\mathrm{O}(1)$ & $0.5577(2)$ & $0.25707(17)$ & $0.25043(19)$ & 0.0342 \\
\hline $\mathrm{O}(2)$ & $0.55516(19)$ & $0.38458(17)$ & $0.06254(17)$ & 0.0325 \\
\hline $\mathrm{C}(2)$ & $0.5445(3)$ & $0.5258(3)$ & $0.0274(3)$ & 0.0329 \\
\hline $\mathrm{C}(3)$ & $0.4657(3)$ & $0.5863(2)$ & $0.1230(3)$ & 0.0271 \\
\hline $\mathrm{C}(4)$ & $0.4784(2)$ & $0.5120(2)$ & $0.3709(2)$ & 0.0223 \\
\hline $\mathrm{O}(3)$ & $0.43455(19)$ & $0.62314(16)$ & $0.38425(18)$ & 0.0312 \\
\hline $\mathrm{C}(5)$ & $0.5129(3)$ & $0.4068(2)$ & $0.4908(2)$ & 0.0226 \\
\hline $\mathrm{C}(6)$ & $0.4137(3)$ & $0.4318(2)$ & $0.5805(2)$ & 0.0244 \\
\hline $\mathrm{C}(7)$ & $0.2493(2)$ & $0.4011(2)$ & $0.4948(2)$ & 0.0243 \\
\hline $\mathrm{C}(8)$ & $0.1386(3)$ & $0.4990(2)$ & $0.4636(3)$ & 0.0287 \\
\hline $\mathrm{C}(9)$ & $-0.0126(3)$ & $0.4667(3)$ & $0.3898(3)$ & 0.0348 \\
\hline $\mathrm{C}(11)$ & $-0.0540(3)$ & $0.3372(3)$ & $0.3447(3)$ & 0.0350 \\
\hline $\mathrm{C}(12)$ & $0.0544(3)$ & $0.2396(3)$ & $0.3727(3)$ & 0.0310 \\
\hline $\mathrm{C}(13)$ & $0.2053(3)$ & $0.2713(2)$ & $0.4482(3)$ & 0.0272 \\
\hline $\mathrm{C}(14)$ & $0.6801(3)$ & $0.4121(2)$ & $0.5833(3)$ & 0.0302 \\
\hline $\mathrm{C}(15)$ & $0.7409(3)$ & $0.4661(3)$ & $0.7147(3)$ & 0.0396 \\
\hline $\mathrm{C}(16)$ & $0.2950(3)$ & $0.6060(2)$ & $0.0437(2)$ & 0.0281 \\
\hline $\mathrm{C}(17)$ & $0.2107(2)$ & $0.4790(2)$ & $-0.0162(2)$ & 0.0253 \\
\hline $\mathrm{C}(18)$ & $0.1577(3)$ & $0.3982(3)$ & $0.0704(2)$ & 0.0289 \\
\hline $\mathrm{C}(19)$ & $0.0875(3)$ & $0.2775(3)$ & $0.0170(3)$ & 0.0341 \\
\hline $\mathrm{C}(20)$ & $0.0674(3)$ & $0.2357(3)$ & $-0.1244(3)$ & 0.0364 \\
\hline & $0.1162(3)$ & $0.3157(3)$ & $-0.2125(3)$ & 0.0361 \\
\hline & $0.1871(3)$ & $0.4362(3)$ & $-0.1599(3)$ & 0.0316 \\
\hline
\end{tabular}

Table 3: Atomic coordinates and isotropic thermal parameters $\left(\AA^{2}\right)$ of hydrogen atoms

\begin{tabular}{|c|c|c|c|c|}
\hline Atom & $\mathbf{x}$ & $\mathrm{y}$ & $\mathrm{Z}$ & $\mathrm{U}_{\text {iso }}$ \\
\hline & & & & \\
\hline $\mathrm{H}(21)$ & 0.6469 & 0.5654 & 0.0525 & 0.0413 \\
\hline $\mathrm{H}(22)$ & 0.4836 & 0.5405 & -0.0796 & 0.0413 \\
\hline $\mathrm{H}(31)$ & 0.4996 & 0.6790 & 0.1566 & 0.0329 \\
\hline $\mathrm{H}(51)$ & 0.4894 & 0.3149 & 0.4489 & 0.0272 \\
\hline $\mathrm{H}(61)$ & 0.4230 & 0.5276 & 0.6107 & 0.0294 \\
\hline $\mathrm{H}(62)$ & 0.4502 & 0.3742 & 0.6705 & 0.0294 \\
\hline $\mathrm{H}(81)$ & 0.1680 & 0.5932 & 0.4943 & 0.0362 \\
\hline $\mathrm{H}(91)$ & -0.0914 & 0.5374 & 0.3695 & 0.0424 \\
\hline $\mathrm{H}(101)$ & -0.1626 & 0.3144 & 0.2916 & 0.0424 \\
\hline $\mathrm{H}(111)$ & 0.0246 & 0.1461 & 0.3390 & 0.0379 \\
\hline$H(121)$ & 0.2834 & 0.1999 & 0.4692 & 0.0343 \\
\hline$H(131)$ & 0.7499 & 0.3709 & 0.5405 & 0.0364 \\
\hline$H(141)$ & 0.8527 & 0.4649 & 0.7677 & 0.0462 \\
\hline$H(142)$ & 0.6755 & 0.5085 & 0.7618 & 0.0462 \\
\hline$H(151)$ & 0.2776 & 0.6691 & -0.0403 & 0.0337 \\
\hline$H(152)$ & 0.2544 & 0.6455 & 0.1153 & 0.0337 \\
\hline$H(171)$ & 0.1704 & 0.4276 & 0.1721 & 0.0351 \\
\hline
\end{tabular}




\begin{tabular}{|c|c|c|c|c|}
\hline $\mathrm{H}(181)$ & 0.0513 & 0.2204 & 0.0809 & 0.0418 \\
\hline $\mathrm{H}(191)$ & 0.0180 & 0.1483 & -0.1622 & 0.0436 \\
\hline $\mathrm{H}(201)$ & 0.1003 & 0.2867 & -0.3152 & 0.0439 \\
\hline $\mathrm{H}(211)$ & 0.2218 & 0.4932 & -0.2250 & 0.0379 \\
\hline
\end{tabular}

Table 4: Anisotropic thermal parameters $\left(\AA^{2}\right)$

\begin{tabular}{|c|l|l|l|l|l|c|}
\hline Atom & $\mathrm{U}_{11}$ & $\mathrm{U}_{22}$ & $\mathrm{U}_{33}$ & $\mathrm{U}_{23}$ & $\mathrm{U}_{13}$ & $\mathrm{U}_{12}$ \\
\hline & & & & & & \\
\hline $\mathrm{N}(1)$ & $0.0268(9)$ & $0.0196(9)$ & $0.0232(9)$ & $0.0004(8)$ & $0.0109(8)$ & $0.0002(8)$ \\
\hline $\mathrm{C}(1)$ & $0.0260(12)$ & $0.0272(13)$ & $0.0254(11)$ & $-0.0033(10)$ & $0.0101(10)$ & $0.0010(10)$ \\
\hline $\mathrm{O}(1)$ & $0.0437(10)$ & $0.0244(9)$ & $0.0365(9)$ & $0.0006(8)$ & $0.0173(8)$ & $0.0090(8)$ \\
\hline $\mathrm{O}(2)$ & $0.0390(10)$ & $0.0357(10)$ & $0.0285(8)$ & $0.0010(8)$ & $0.0190(7)$ & $0.0062(8)$ \\
\hline $\mathrm{C}(2)$ & $0.0357(14)$ & $0.0381(14)$ & $0.0295(13)$ & $0.0010(11)$ & $0.0176(11)$ & $-0.0027(12)$ \\
\hline $\mathrm{C}(3)$ & $0.0327(12)$ & $0.0231(12)$ & $0.0264(11)$ & $0.0042(10)$ & $0.0120(10)$ & $-0.0015(10)$ \\
\hline $\mathrm{C}(4)$ & $0.0225(11)$ & $0.0222(12)$ & $0.0234(11)$ & $-0.0045(9)$ & $0.0101(9)$ & $-0.0043(9)$ \\
\hline $\mathrm{O}(3)$ & $0.0443(10)$ & $0.0210(9)$ & $0.0300(9)$ & $-0.0015(7)$ & $0.0158(8)$ & $0.0021(8)$ \\
\hline $\mathrm{C}(5)$ & $0.0265(11)$ & $0.0176(10)$ & $0.0240(10)$ & $0.0020(9)$ & $0.0097(8)$ & $0.0003(9)$ \\
\hline $\mathrm{C}(6)$ & $0.0297(11)$ & $0.0234(12)$ & $0.0205(10)$ & $0.0000(9)$ & $0.0098(9)$ & $-0.0009(10)$ \\
\hline $\mathrm{C}(7)$ & $0.0282(11)$ & $0.0279(12)$ & $0.0201(10)$ & $-0.0002(9)$ & $0.0129(9)$ & $-0.0010(10)$ \\
\hline $\mathrm{C}(8)$ & $0.0372(13)$ & $0.0235(12)$ & $0.0298(12)$ & $0.0013(10)$ & $0.0176(10)$ & $0.0043(10)$ \\
\hline $\mathrm{C}(9)$ & $0.0342(13)$ & $0.0391(15)$ & $0.0328(13)$ & $0.0064(12)$ & $0.0143(11)$ & $0.0091(12)$ \\
\hline $\mathrm{C}(10)$ & $0.0266(13)$ & $0.0497(17)$ & $0.0298(13)$ & $-0.0008(12)$ & $0.0118(11)$ & $-0.0019(12)$ \\
\hline $\mathrm{C}(11)$ & $0.0320(13)$ & $0.0314(14)$ & $0.0314(13)$ & $-0.0008(11)$ & $0.0139(11)$ & $-0.0040(11)$ \\
\hline $\mathrm{C}(12)$ & $0.0308(12)$ & $0.0263(12)$ & $0.0285(12)$ & $0.0013(10)$ & $0.0156(10)$ & $0.0011(10)$ \\
\hline $\mathrm{C}(13)$ & $0.0287(12)$ & $0.0285(13)$ & $0.0339(12)$ & $0.0060(11)$ & $0.0124(10)$ & $0.0008(11)$ \\
\hline $\mathrm{C}(14)$ & $0.0347(13)$ & $0.0459(16)$ & $0.0350(14)$ & $-0.0003(13)$ & $0.0091(11)$ & $-0.0069(13)$ \\
\hline $\mathrm{C}(15)$ & $0.0338(13)$ & $0.0265(12)$ & $0.0239(11)$ & $0.0032(10)$ & $0.0107(10)$ & $0.0034(11)$ \\
\hline $\mathrm{C}(16)$ & $0.0223(10)$ & $0.0299(13)$ & $0.0235(11)$ & $0.0012(10)$ & $0.0083(9)$ & $0.0034(10)$ \\
\hline $\mathrm{C}(17)$ & $0.0308(12)$ & $0.0349(13)$ & $0.0220(11)$ & $0.0008(11)$ & $0.0111(9)$ & $-0.0005(11)$ \\
\hline $\mathrm{C}(18)$ & $0.0360(13)$ & $0.0379(15)$ & $0.0307(13)$ & $0.0023(12)$ & $0.0150(11)$ & $-0.0055(12)$ \\
\hline $\mathrm{C}(19)$ & $0.0373(14)$ & $0.0395(16)$ & $0.0323(13)$ & $-0.0070(12)$ & $0.0127(11)$ & $-0.0089(12)$ \\
\hline $\mathrm{C}(20)$ & $0.0392(14)$ & $0.0446(16)$ & $0.0261(13)$ & $-0.0059(11)$ & $0.0140(11)$ & $-0.0061(13)$ \\
\hline $\mathrm{C}(21)$ & $0.0320(12)$ & $0.0408(15)$ & $0.0218(11)$ & $0.0040(11)$ & $0.0097(10)$ & $0.0007(11)$ \\
\hline
\end{tabular}

Table 5: Bond lengths $(\AA)$

\begin{tabular}{|c|c|c|c|}
\hline $\mathrm{N}(1)-\mathrm{C}(1)$ & $\mathrm{C}(7)-\mathrm{C}(12)$ & $1.393(3)$ \\
\hline $\mathrm{N}(1)-\mathrm{C}(3)$ & $1.387(3)$ & $\mathrm{C}(8)-\mathrm{C}(9)$ & $1.393(3)$ \\
\hline $\mathrm{N}(1)-\mathrm{C}(4)$ & $1.476(3)$ & $\mathrm{C}(9)-\mathrm{C}(10)$ & $1.382(4)$ \\
\hline $\mathrm{C}(1)-\mathrm{O}(1)$ & $1.403(3)$ & $\mathrm{C}(10)-\mathrm{C}(11)$ & $1.378(4)$ \\
\hline $\mathrm{C}(1)-\mathrm{O}(2)$ & $1.200(3)$ & $\mathrm{C}(11)-\mathrm{C}(12)$ & $1.392(3)$ \\
\hline $\mathrm{O}(2)-\mathrm{C}(2)$ & $1.350(3)$ & $\mathrm{C}(13)-\mathrm{C}(14)$ & $1.313(4)$ \\
\hline $\mathrm{C}(2)-\mathrm{C}(3)$ & $1.453(3)$ & $\mathrm{C}(15)-\mathrm{C}(16)$ & $1.505(3)$ \\
\hline $\mathrm{C}(3)-\mathrm{C}(15)$ & $1.528(3)$ & $\mathrm{C}(16)-\mathrm{C}(17)$ & $1.397(3)$ \\
\hline $\mathrm{C}(4)-\mathrm{O}(3)$ & $1.538(3)$ & $\mathrm{C}(16)-\mathrm{C}(21)$ & $1.404(3)$ \\
\hline $\mathrm{C}(4)-\mathrm{C}(5)$ & $1.215(3)$ & $\mathrm{C}(17)-\mathrm{C}(18)$ & $1.389(4)$ \\
\hline $\mathrm{C}(5)-\mathrm{C}(6)$ & $1.519(3)$ & $\mathrm{C}(18)-\mathrm{C}(19)$ & $1.387(3)$ \\
\hline $\mathrm{C}(5)-\mathrm{C}(13)$ & $1.535(3)$ & $\mathrm{C}(19)-\mathrm{C}(20)$ & $1.379(4)$ \\
\hline $\mathrm{C}(6)-\mathrm{C}(7)$ & $1.517(3)$ & $\mathrm{C}(20)-\mathrm{C}(21)$ & $1.389(4)$ \\
\hline $\mathrm{C}(7)-\mathrm{C}(8)$ & $1.513(3)$ & & \\
\hline
\end{tabular}

Note $-\mathrm{H}$ atoms have been excluded 
Table 6: Bond angles $\left({ }^{\circ}\right)$

\begin{tabular}{|c|c|c|c|}
\hline $\mathrm{C}(1)-\mathrm{N}(1)-\mathrm{C}(3)$ & $110.75(18)$ & $\mathrm{C}(6)-\mathrm{C}(7)-\mathrm{C}(8)$ & $121.8(2)$ \\
\hline $\mathrm{C}(1)-\mathrm{N}(1)-\mathrm{C}(4)$ & $128.73(19)$ & $\mathrm{C}(6)-\mathrm{C}(7)-\mathrm{C}(12)$ & $119.8(2)$ \\
\hline $\mathrm{C}(3)-\mathrm{N}(1)-\mathrm{C}(4)$ & $119.96(18)$ & $\mathrm{C}(8)-\mathrm{C}(7)-\mathrm{C}(12)$ & $118.4(2)$ \\
\hline $\mathrm{N}(1)-\mathrm{C}(1)-\mathrm{O}(1)$ & $129.3(2)$ & $\mathrm{C}(7)-\mathrm{C}(8)-\mathrm{C}(9)$ & $120.5(2)$ \\
\hline $\mathrm{N}(1)-\mathrm{C}(1)-\mathrm{O}(2)$ & $108.7(2)$ & $\mathrm{C}(8)-\mathrm{C}(9)-\mathrm{C}(10)$ & $120.2(2)$ \\
\hline $\mathrm{O}(1)-\mathrm{C}(1)-\mathrm{O}(2)$ & $122.0(2)$ & $\mathrm{C}(9)-\mathrm{C}(10)-\mathrm{C}(11)$ & $120.0(2)$ \\
\hline $\mathrm{C}(1)-\mathrm{O}(2)-\mathrm{C}(2)$ & $110.23(18)$ & $\mathrm{C}(10)-\mathrm{C}(11)-\mathrm{C}(12)$ & $119.9(2)$ \\
\hline $\mathrm{O}(2)-\mathrm{C}(2)-\mathrm{C}(3)$ & $104.50(18)$ & $\mathrm{C}(7)-\mathrm{C}(12)-\mathrm{C}(11)$ & $121.0(2)$ \\
\hline $\mathrm{N}(1)-\mathrm{C}(3)-\mathrm{C}(2)$ & $99.99(19)$ & $\mathrm{C}(5)-\mathrm{C}(13)-\mathrm{C}(14)$ & $125.6(2)$ \\
\hline $\mathrm{N}(1)-\mathrm{C}(3)-\mathrm{C}(15)$ & $112.24(18)$ & $\mathrm{C}(3)-\mathrm{C}(15)-\mathrm{C}(16)$ & $113.67(19)$ \\
\hline $\mathrm{C}(2)-\mathrm{C}(3)-\mathrm{C}(15)$ & $114.6(2)$ & $\mathrm{C}(15)-\mathrm{C}(16)-\mathrm{C}(17)$ & $121.29(19)$ \\
\hline $\mathrm{N}(1)-\mathrm{C}(4)-\mathrm{O}(3)$ & $117.2(2)$ & $\mathrm{C}(15)-\mathrm{C}(16)-\mathrm{C}(21)$ & $120.7(2)$ \\
\hline $\mathrm{N}(1)-\mathrm{C}(4)-\mathrm{C}(5)$ & $120.12(19)$ & $\mathrm{C}(17)-\mathrm{C}(16)-\mathrm{C}(21)$ & $117.9(2)$ \\
\hline $\mathrm{O}(3)-\mathrm{C}(4)-\mathrm{C}(5)$ & $122.66(19)$ & $\mathrm{C}(16)-\mathrm{C}(17)-\mathrm{C}(18)$ & $120.9(2)$ \\
\hline $\mathrm{C}(4)-\mathrm{C}(5)-\mathrm{C}(6)$ & $108.88(18)$ & $\mathrm{C}(17)-\mathrm{C}(18)-\mathrm{C}(19)$ & $120.4(2)$ \\
\hline $\mathrm{C}(4)-\mathrm{C}(5)-\mathrm{C}(13)$ & $108.26(18)$ & $\mathrm{C}(18)-\mathrm{C}(19)-\mathrm{C}(20)$ & $119.5(2)$ \\
\hline $\mathrm{C}(6)-\mathrm{C}(5)-\mathrm{C}(13)$ & $113.19(18)$ & $\mathrm{C}(19)-\mathrm{C}(20)-\mathrm{C}(21)$ & $120.5(2)$ \\
\hline $\mathrm{C}(5)-\mathrm{C}(6)-\mathrm{C}(7)$ & $112.81(17)$ & $\mathrm{C}(16)-\mathrm{C}(21)-\mathrm{C}(20)$ & $120.8(2)$ \\
\hline
\end{tabular}

Note $-\mathrm{H}$ atoms have been excluded 
-2-one (1)

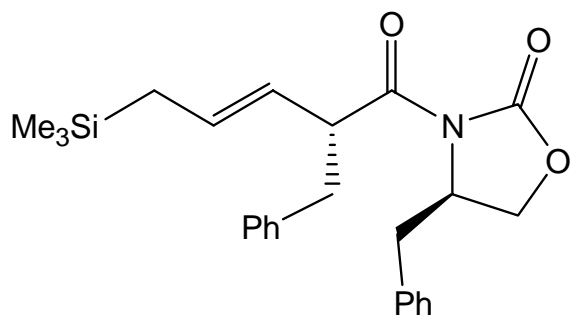

To a solution of 4 (3.42 mmol, $1.10 \mathrm{~g})$ and allyltrimethylsilane (10.26 mmol, 1.63 $\mathrm{mL})$ in DCM (50 mL) at reflux was added $5 \mathrm{~mol} \%$ of Grubbs' second generation catalyst (145 mg). After $68 \mathrm{hrs}$ the solvent was removed in vacuo. The crude product was purified by column chromatography to yield a white solid. $(1.37 \mathrm{~g}, 95 \%) ;{ }^{1} \mathrm{H}$ NMR (400 MHz, $\left.\mathrm{CDCl}_{3}\right): \delta=7.0-7.32(\mathrm{~m}, 10 \mathrm{H}), 5.62(\mathrm{dt}, J=8.1,15.2,1 \mathrm{H}), 5.29-5.36$ $(\mathrm{m}, 1 \mathrm{H}), 4.82(\mathrm{~m}, 1 \mathrm{H}), 4.61(\mathrm{~m}, 1 \mathrm{H}), 4.01-4.15(\mathrm{~m}, 2 \mathrm{H}), 3.22(\mathrm{dd}, J=8.3,13.4,1 \mathrm{H})$, $3.02(\mathrm{dd}, J=3.3,13.3,1 \mathrm{H}), 2.85(\mathrm{dd}, J=6.1,13.4,1 \mathrm{H}), 2.57(\mathrm{dd}, J=9.1,13.4,1 \mathrm{H})$ $1.42(\mathrm{~d}, J=8.1,1 \mathrm{H})-0.08(\mathrm{~s}, 9 \mathrm{H}) ;{ }^{13} \mathrm{C} \mathrm{NMR}\left(100 \mathrm{MHz}, \mathrm{CDCl}_{3}\right): \delta=174.2,138.9$, $135.1,131.6,129.4,128.9,128.3,127.2,126.3,124.9,65.7,55.2,48.2,38.9,37.6$, 23.1, -2.1; IR $\left(\mathrm{CHCl}_{3}\right): v 3444,2955,1767,1694,1454,1393,1248,1182 \mathrm{~cm}^{-1}$; MS $\left(\mathrm{CI}\left(\mathrm{NH}_{3}\right)\right): m / z 422\left(\mathrm{M}+\mathrm{H}^{+}\right) ; \mathrm{HMRS}$ required for $\mathrm{C}_{25} \mathrm{H}_{32} \mathrm{NO}_{3} \mathrm{Si}\left([\mathrm{M}]^{+}\right) 422.2158$ found

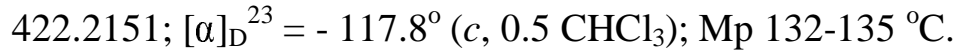

\section{(2R)-2-benzyl-5-(trimethylsilyl)pent-3-enoic acid (6)}<smiles>C[SiH3]C/C=C/[C@H](Cc1ccccc1)C(=O)O</smiles>

To a solution of (2R)-2-benzylbut-3-enoic acid (1.4 mmol, $250 \mathrm{mg})$ and allyltrimethylsilane $(4.2 \mathrm{mmol}, 0.67 \mathrm{~mL})$ in $\mathrm{DCM}(3 \mathrm{~mL})$ at reflux was added 
Hoveyda-Grubbs second generation catalyst (5 mol\%, $44 \mathrm{mg}$ ). The reaction was allowed to stir at reflux for $50 \mathrm{hrs}$. The solvent was removed in vacuo. The crude product was purified by column chromatography (184 mg, 50\%). ${ }^{1} \mathrm{H}$ NMR (400 $\left.\mathrm{MHz}, \mathrm{CDCl}_{3}\right): \delta=7.15-7.30(\mathrm{~m}, 5 \mathrm{H}), 5.48-5.56(\mathrm{~m}, 1 \mathrm{H}), 5.26(\mathrm{dd}, J=8.8,15.2,1 \mathrm{H})$, $3.30(\mathrm{~m}, 1 \mathrm{H}), 3.10(\mathrm{dd}, J=7.1,13.9,1 \mathrm{H}), 2.80(\mathrm{dd}, J=7.8,13.6,1 \mathrm{H}), 1.43(\mathrm{~d}, J=8.3$ 2H), $-0.08(\mathrm{~s}, 9 \mathrm{H}) ;{ }^{13} \mathrm{C}$ NMR $\left(125 \mathrm{MHz}, \mathrm{CDCl}_{3}\right): \delta=179.5,138.7,131.4,130.1$, $129.1,129.0,128.3,126.4,126.3,124.3,123.7,50.8,38.5,22.9,18.9,-2.08$; IR $\left(\mathrm{CHCl}_{3}\right): v$ 2955, 1797; HMRS required for $\mathrm{C}_{15} \mathrm{H}_{21} \mathrm{O}_{2} \mathrm{Si}\left([\mathrm{M}-\mathrm{H}]^{-}\right) 261.1311$ found $261.1318 ;[\alpha]_{\mathrm{D}}^{21}=-39.4\left(c, 0.5, \mathrm{CHCl}_{3}\right)$.

(4R)-4-benzyl-3-[(2S, 3R)-2-benzyl-3-fluoropent-4-enoyl]-1,3-oxazolidin-2-one (7)

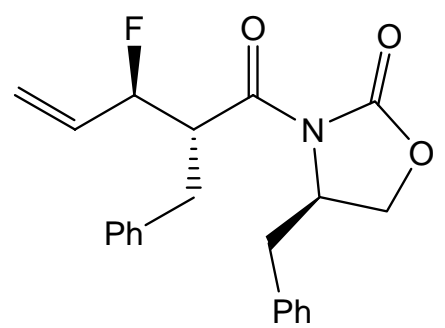

To a solution of $1(0.88 \mathrm{mmol}, 370 \mathrm{mg})$ in $\mathrm{CH}_{3} \mathrm{CN}(8 \mathrm{~mL})$ was added Selectfluor (0.88 mmol, $310 \mathrm{mg})$. The reaction was allowed to stir at RT for $48 \mathrm{hr}$. The solvent was removed in vacuo. The crude product was purified by column chromatography to yield a mixture of diastereoisomers $d e=10 \%(250 \mathrm{mg}, 95 \%) .{ }^{1} \mathrm{H}$ NMR $(400 \mathrm{MHz}$, $\left.\mathrm{CDCl}_{3}\right): \delta=6.98-7.3(\mathrm{~m}, 10 \mathrm{H}), 6.0(\mathrm{~m}, 1 \mathrm{H}), 5.5(\mathrm{dm}, J=17,1 \mathrm{H}), 5.42(\mathrm{~d}, J=10.6,1 \mathrm{H})$ 5.1 (ddd, $J=7.1,8.1,47.2,1 \mathrm{H}), 4.85(\mathrm{~m}, 1 \mathrm{H}), 4.64(\mathrm{~m}, 1 \mathrm{H}), 3.98-4.12(\mathrm{~m}, 2 \mathrm{H}), 2.84-$ $3.0(\mathrm{~m}, 3 \mathrm{H}), 2.2(\mathrm{~m}, 1 \mathrm{H}) ;{ }^{13} \mathrm{C} \mathrm{NMR}\left(100 \mathrm{MHz}, \mathrm{CDCl}_{3}\right): \delta=172.5,152.9,137.2$ $135.1,133.7,133.6,129.5,129.3,128.9,128.5,127.2,126.8,120.6,120.5,94.4$ (d, $J=175), 65.6,55.1,48.1(\mathrm{~d}, J=21), 37.334 .8(\mathrm{~d}, J=6) ;{ }^{19} \mathrm{~F}\left\{{ }^{1} \mathrm{H}\right\}$ NMR $(376.5 \mathrm{MHz}$, $\left.\mathrm{CDCl}_{3}\right): \delta=-175.7 ; \mathrm{IR}\left(\mathrm{CHCl}_{3}\right): v 3444,2957,1645,1367,1235,1205 \mathrm{~cm}^{-1} ;[\alpha]_{\mathrm{D}}{ }^{20}=-$ 
$18.4^{\mathrm{o}}\left(c, 0.5 \mathrm{CHCl}_{3}\right)$; HMRS required for $\mathrm{C}_{22} \mathrm{H}_{23} \mathrm{NO}_{3} \mathrm{~F} 368.1662\left(\left[\mathrm{M}+\mathrm{H}^{+}\right]\right)$found 368.1669 .

(4R)-4-benzyl-3-[(2S, 3S)-2-benzyl-3-fluoropent-4-enoyl]-1,3-oxazolidin-2-one (7)

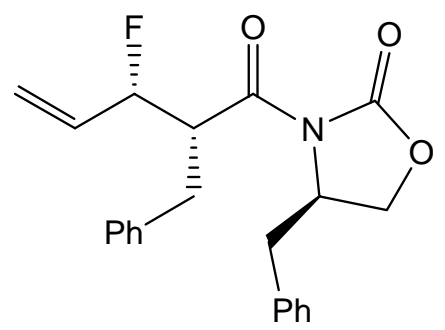

${ }^{1} \mathrm{H}$ NMR $\left(400 \mathrm{MHz}, \mathrm{CDCl}_{3}\right): \delta=6.92-7.31(\mathrm{~m}, 10 \mathrm{H}), 6.05(\mathrm{dddd}, J=6.5,10.6,14.0$, $17.2,1 \mathrm{H}), 5.41-5.46(\mathrm{~m}, 1 \mathrm{H}), 5.35-5.38(\mathrm{~m}, 1 \mathrm{H}), 5.15(\mathrm{dt}, J=6.4,47.7,1 \mathrm{H}), 4.75-$ $4.84(\mathrm{~m}, 1 \mathrm{H}), 4.59-4.64(\mathrm{~m}, 1 \mathrm{H}), 4.11(\mathrm{~m}, 1 \mathrm{H}), 4.03(\mathrm{dd}, J=2.7,9.1,1 \mathrm{H}), 3.18-3.15$ $(\mathrm{m}, 2 \mathrm{H}), 2.90(\mathrm{dd}, J=3.3,13.5,1 \mathrm{H}), 2.33(\mathrm{dd}, J=9.2,13.5,1 \mathrm{H}) ;{ }^{13} \mathrm{C}$ NMR $(100$ $\left.\mathrm{MHz}, \mathrm{CDCl}_{3}\right): \delta=171.6(\mathrm{~d}, J=6.7), 153.0,138.0,134.9,134.2(\mathrm{~d}, J=20.2), 129.3$, $129.2,128.9,128.4,127.2,126.5,119.4$ (d, $J=12.1), 94.5$ (d, $J=175.2), 65.7,55.0$, $49.0(\mathrm{~d}, J=23.3), 37.2,33.6(\mathrm{~d}, J=4.6) ;{ }^{19} \mathrm{~F} \mathrm{NMR}\left(376.5 \mathrm{MHz}, \mathrm{CDCl}_{3}\right): \delta-184.3$ $\left(\mathrm{dtd}, J_{\mathrm{HF}}=2.8,14.7,47.7\right)$. MS ESI m/z $368[\mathrm{M}+\mathrm{H}]^{+}$HR-MS Calc for $\mathrm{C}_{22} \mathrm{H}_{22} \mathrm{NO}_{3} \mathrm{~F}$ 368.1662, Found 368.1656, $[\alpha]_{\mathrm{D}}^{20}=-7.8\left(\mathrm{c}=1, \mathrm{CHCl}_{3}\right)$. 


\section{Single-crystal X-ray diffraction report for (4R)-4-benzyl-3-[(2S, $3 R$ )-2-benzyl-3-fluoropent-4-enoyl]-1,3-oxazolidin-2-one, $\mathrm{C}_{22} \mathrm{H}_{22} \mathrm{FNO}_{3}$}

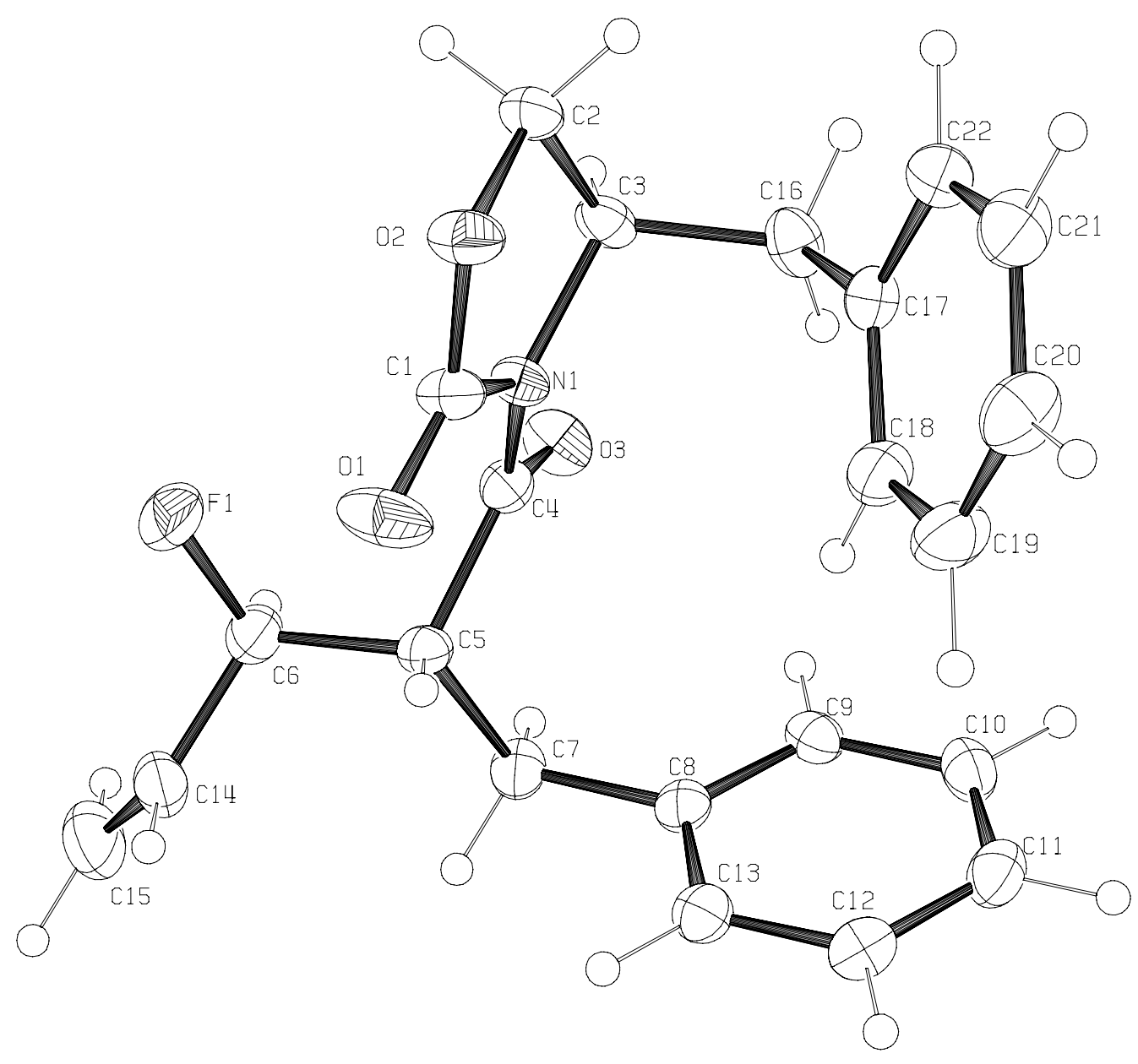

Crystals of (4R)-4-benzyl-3-[(2S, 3R)-2-benzyl-3-fluoropent-4-enoyl]1,3-oxazolidin-2-one were grown by recrystallisation from ethyl acetate. A single crystal having dimensions approximately $0.06 \times 0.08 \times 0.40 \mathrm{~mm}$ was mounted on a glass fibre using perfluoropolyether oil and cooled rapidly to $150 \mathrm{~K}$ in a stream of cold $\mathrm{N}_{2}$ using an Oxford Cryosystems CRYOSTREAM unit. Diffraction data were measured using an Enraf-Nonius KappaCCD diffractometer (graphite-monochromated $\mathrm{MoK}_{\alpha}$ radiation, $\lambda=0.71073 \AA$ ). Intensity data were processed using the DENZO-SMN package ${ }^{1}$.

Examination of the systematic absences of the intensity data showed the space group

to

be $\begin{array}{llll}P & 2 & 2 & 2\end{array} 2_{1}$. The structure was solved using the direct-methods program SIR92 ${ }^{2}$, which located all non-hydrogen atoms. Subsequent full-matrix leastsquares refinement was carried out using the CRYSTALS program suite ${ }^{3}$. 
Coordinates and anisotropic thermal parameters of all non-hydrogen atoms were refined. Hydrogen atoms were positioned geometrically after each cycle of refinement. A 3-term Chebychev polynomial weighting scheme was applied. Refinement converged satisfactorily to give $R=0.0330, w R=$ 0.0372 .

Attached is a thermal ellipsoid plot (ORTEP- $3^{4}$ ) at $40 \%$ probability. A summary of crystallographic data is given below, as are full lists of atomic coordinates, anisotropic thermal parameters and those bond lengths and angles not concerning $\mathrm{H}$ atoms.

\section{References:}

1 Z. Otwinowski and W. Minor, Processing of X-ray Diffraction Data Collected in Oscillation Mode, Methods Enzymol., 1997, 276, Eds C. W. Carter and R. M. Sweet, Academic Press.

2 A. Altomare, G. Cascarano, G. Giacovazzo, A. Guagliardi ,M. C. Burla, G. Polidori and M. Camalli, J. Appl. Cryst. 1994, 27, 435.

3 CRYSTALS Issue 12, P. W. Betteridge, J. R. Cooper, R. I. Cooper, K. Prout and D. J. Watkin, J. Appl. Cryst., 2003, 36, 1487

$4 \quad$ ORTEP-3 v. 1.0.2, C. K. Johnson and M. K. Burnett, 1998.

Table 1: Crystal data and refinement details

\begin{tabular}{|c|c|}
\hline Crystal identification & ARC986 \\
\hline Chemical formula & $\mathrm{C}_{22} \mathrm{H}_{22} \mathrm{FNO}_{3}$ \\
\hline Formula weight & 367.42 \\
\hline Temperature $(\mathrm{K})$ & 150 \\
\hline Wavelength $(\AA)$ & 0.71073 \\
\hline Crystal system & Orthorhombic \\
\hline Space group & $P 2_{1} 2_{1} 2_{1}$ \\
\hline$a(\AA)$ & $6.3654(2)$ \\
\hline$b(\dot{A})$ & 17.2322(5) \\
\hline$c(\AA)$ & $17.8046(7)$ \\
\hline$\alpha\left(^{\circ}\right)$ & 90 \\
\hline$\beta\left(^{\circ}\right)$ & 90 \\
\hline$\gamma\left({ }^{\circ}\right)$ & 90 \\
\hline Cell volume $\left(\AA^{3}\right)$ & 1952.98(11) \\
\hline Z & 4 \\
\hline Calculated density $\left(\mathrm{Mg} / \mathrm{m}^{3}\right)$ & 1.250 \\
\hline Absorption coefficient $\left(\mathrm{mm}^{-1}\right)$ & 0.089 \\
\hline $\mathrm{F}_{000}$ & 776 \\
\hline Crystal size $(\mathrm{mm})$ & $0.06 \times 0.08 \times 0.40$ \\
\hline Description of crystal & Colourless needle \\
\hline Absorption correction & Semi-empirical from equivalent reflections \\
\hline Transmission coefficients (min,max) & $0.97,0.99$ \\
\hline$\theta$ range for data collection $\left({ }^{\circ}\right)$ & $5.0 \leq \theta \leq 27.5$ \\
\hline Index ranges & $-8 \leq h \leq 8,-22 \leq k \leq 22,-23 \leq I \leq 23$ \\
\hline Reflections measured & 12113 \\
\hline Unique reflections & 2562 \\
\hline
\end{tabular}




\begin{tabular}{|l|l|}
\hline$R_{\text {int }}$ & 0.051 \\
\hline Observed reflections $(I>3 \sigma(I))$ & 1658 \\
\hline Refinement method & Full-matrix least-squares on $F$ \\
\hline Parameters refined & 244 \\
\hline Weighting scheme & Chebychev 3-term polynomial \\
\hline Goodness of fit & 1.1252 \\
\hline$R$ & 0.0330 \\
\hline wR & 0.0372 \\
\hline Residual electron density (min,max) $\left(\mathrm{e}^{-3}\right)$ & $-0.14,0.17$ \\
\hline
\end{tabular}

Table 2: Atomic coordinates and equivalent isotropic thermal parameters $\left(\AA^{2}\right)$ of non-hydrogen atoms

\begin{tabular}{|c|c|c|c|c|}
\hline Atom & $\mathrm{X}$ & $\mathrm{y}$ & $\mathrm{Z}$ & $\mathrm{U}_{\text {equiv }}$ \\
\hline & & & & \\
\hline $\mathrm{N}(1)$ & $0.5550(3)$ & $0.25107(11)$ & $0.19887(10)$ & 0.0248 \\
\hline $\mathrm{C}(1)$ & $0.4048(4)$ & $0.23078(15)$ & $0.14541(13)$ & 0.0299 \\
\hline $\mathrm{O}(1)$ & $0.2188(3)$ & $0.22436(13)$ & $0.15300(10)$ & 0.0465 \\
\hline $\mathrm{O}(2)$ & $0.5016(3)$ & $0.21865(10)$ & $0.07938(9)$ & 0.0349 \\
\hline $\mathrm{C}(2)$ & $0.7285(4)$ & $0.22188(14)$ & $0.08858(14)$ & 0.0331 \\
\hline $\mathrm{C}(3)$ & $0.7618(4)$ & $0.26370(15)$ & $0.16283(13)$ & 0.0294 \\
\hline $\mathrm{C}(4)$ & $0.5383(4)$ & $0.25508(13)$ & $0.27653(12)$ & 0.0269 \\
\hline $\mathrm{O}(3)$ & $0.6958(3)$ & $0.26618(11)$ & $0.31313(9)$ & 0.0383 \\
\hline $\mathrm{C}(5)$ & $0.3246(4)$ & $0.24459(13)$ & $0.31433(13)$ & 0.0271 \\
\hline $\mathrm{C}(6)$ & $0.3095(5)$ & $0.16091(14)$ & $0.34391(14)$ & 0.0352 \\
\hline $\mathrm{F}(1)$ & $0.3351(3)$ & $0.11143(9)$ & $0.28149(10)$ & 0.0583 \\
\hline $\mathrm{C}(8)$ & $0.3057(4)$ & $0.30221(13)$ & $0.38021(13)$ & 0.0321 \\
\hline $\mathrm{C}(9)$ & $0.2934(4)$ & $0.38562(13)$ & $0.35332(12)$ & 0.0285 \\
\hline $\mathrm{C}(10)$ & $0.4652(4)$ & $0.43498(14)$ & $0.35724(13)$ & 0.0312 \\
\hline $\mathrm{C}(11)$ & $0.4508(5)$ & $0.51091(14)$ & $0.33038(14)$ & 0.0358 \\
\hline $\mathrm{C}(13)$ & $0.2660(5)$ & $0.53704(14)$ & $0.29869(14)$ & 0.0380 \\
\hline $\mathrm{C}(14)$ & $0.0937(5)$ & $0.48834(14)$ & $0.29472(14)$ & 0.0355 \\
\hline $\mathrm{C}(15)$ & $0.1067(4)$ & $0.41278(14)$ & $0.32225(13)$ & 0.0325 \\
\hline $\mathrm{C}(16)$ & $0.1050(5)$ & $0.14302(15)$ & $0.37936(15)$ & 0.0396 \\
\hline $\mathrm{C}(17)$ & $0.0883(6)$ & $0.11249(18)$ & $0.44652(18)$ & 0.0566 \\
\hline $\mathrm{C}(19)$ & $0.8123(4)$ & $0.35050(15)$ & $0.15569(14)$ & 0.0343 \\
\hline $\mathrm{C}(20)$ & $0.6646(4)$ & $0.39511(13)$ & $0.10565(13)$ & 0.0283 \\
\hline $\mathrm{C}(21)$ & $0.4769(5)$ & $0.42471(14)$ & $0.13341(14)$ & 0.0358 \\
\hline $\mathrm{C}(22)$ & $0.3370(5)$ & $0.46305(16)$ & $0.08697(16)$ & 0.0445 \\
\hline & $0.3840(5)$ & $0.47314(16)$ & $0.01135(15)$ & 0.0438 \\
\hline & $0.5715(5)$ & $0.44558(15)$ & $-0.01632(14)$ & 0.0414 \\
\hline & $0.7118(4)$ & $0.40715(14)$ & $0.03009(14)$ & 0.0351 \\
\hline
\end{tabular}

Table 3: Atomic coordinates and isotropic thermal parameters $\left(\AA^{2}\right)$ of hydrogen atoms

\begin{tabular}{|c|c|c|c|c|}
\hline Atom & $\mathrm{x}$ & $\mathrm{y}$ & $\mathrm{z}$ & $\mathrm{U}_{\text {iso }}$ \\
\hline & & & & \\
\hline $\mathrm{H}(21)$ & 0.7946 & 0.2515 & 0.0464 & 0.0397 \\
\hline $\mathrm{H}(22)$ & 0.7893 & 0.1684 & 0.0905 & 0.0397 \\
\hline $\mathrm{H}(31)$ & 0.8865 & 0.2438 & 0.1911 & 0.0353 \\
\hline $\mathrm{H}(51)$ & 0.2086 & 0.2546 & 0.2777 & 0.0325 \\
\hline $\mathrm{H}(61)$ & 0.4197 & 0.1537 & 0.3834 & 0.0423 \\
\hline $\mathrm{H}(71)$ & 0.4315 & 0.2964 & 0.4134 & 0.0385 \\
\hline
\end{tabular}




\begin{tabular}{|c|c|c|c|c|}
\hline$H(72)$ & 0.1758 & 0.2899 & 0.4094 & 0.0385 \\
\hline$H(91)$ & 0.6001 & 0.4161 & 0.3794 & 0.0374 \\
\hline$H(101)$ & 0.5746 & 0.5464 & 0.3341 & 0.0429 \\
\hline$H(111)$ & 0.2568 & 0.5911 & 0.2786 & 0.0456 \\
\hline$H(121)$ & -0.0405 & 0.5074 & 0.2720 & 0.0426 \\
\hline$H(131)$ & -0.0187 & 0.3779 & 0.3197 & 0.0390 \\
\hline$H(141)$ & -0.0265 & 0.1548 & 0.3508 & 0.0476 \\
\hline$H(151)$ & 0.2176 & 0.1002 & 0.4762 & 0.0680 \\
\hline$H(152)$ & -0.0536 & 0.1014 & 0.4681 & 0.0680 \\
\hline$H(161)$ & 0.8069 & 0.3740 & 0.2070 & 0.0412 \\
\hline$H(162)$ & 0.9575 & 0.3557 & 0.1349 & 0.0412 \\
\hline$H(181)$ & 0.4425 & 0.4182 & 0.1878 & 0.0430 \\
\hline$H(201)$ & 0.2020 & 0.4835 & 0.1078 & 0.0534 \\
\hline$H(211)$ & 0.2826 & 0.5002 & -0.0226 & 0.0526 \\
\hline$H(221)$ & 0.6068 & 0.4534 & -0.0705 & 0.0496 \\
\hline & 0.8480 & 0.3879 & 0.0092 & 0.0421 \\
\hline
\end{tabular}

Table 4: Anisotropic thermal parameters $\left(\AA^{2}\right)$

\begin{tabular}{|c|c|c|c|c|c|c|}
\hline Atom & $U_{11}$ & $U_{22}$ & $U_{33}$ & $U_{23}$ & $U_{13}$ & $U_{12}$ \\
\hline$N(1)$ & $0.0165(9)$ & $0.0317(10)$ & $0.0261(9)$ & $0.0002(8)$ & $-0.0006(7)$ & $0.0000(8)$ \\
\hline $\mathrm{C}(1)$ & $0.0237(12)$ & $0.0384(13)$ & $0.0276(12)$ & $-0.0078(10)$ & $0.0011(10)$ & $0.0020(11)$ \\
\hline $\mathrm{O}(1)$ & $0.0194(9)$ & $0.0802(15)$ & $0.0399(10)$ & $-0.0153(10)$ & $0.0001(8)$ & $-0.0042(10)$ \\
\hline $\mathrm{O}(2)$ & $0.0282(9)$ & $0.0474(10)$ & $0.0292(8)$ & $-0.0096(8)$ & $0.0034(8)$ & $0.0007(8)$ \\
\hline $\mathrm{C}(2)$ & $0.0244(12)$ & $0.0347(12)$ & $0.0402(13)$ & $0.0017(11)$ & $0.0080(11)$ & $0.0029(11)$ \\
\hline$C(3)$ & $0.0187(11)$ & $0.0360(12)$ & $0.0335(12)$ & $0.0067(10)$ & $0.0026(10)$ & $0.0020(10)$ \\
\hline $\mathrm{C}(4)$ & $0.0273(12)$ & $0.0257(11)$ & $0.0277(11)$ & $0.0013(9)$ & $-0.0015(10)$ & $0.0026(10)$ \\
\hline $\mathrm{O}(3)$ & $0.0302(9)$ & $0.0515(11)$ & $0.0332(9)$ & $0.0028(8)$ & $-0.0086(8)$ & $-0.0040(9)$ \\
\hline$C(5)$ & $0.0280(12)$ & $0.0281(11)$ & $0.0252(11)$ & $-0.0019(9)$ & $0.0035(10)$ & $0.0018(10)$ \\
\hline$C(6)$ & $0.0428(16)$ & $0.0287(12)$ & $0.0342(13)$ & $-0.0019(10)$ & $0.0056(13)$ & $0.0001(12)$ \\
\hline$F(1)$ & $0.0821(14)$ & $0.0341(8)$ & $0.0586(10)$ & $-0.0135(7)$ & $0.0322(10)$ & $-0.0050(9)$ \\
\hline$C(7)$ & $0.0442(15)$ & $0.0296(12)$ & $0.0225(11)$ & $-0.0021(9)$ & $0.0031(12)$ & $-0.0030(12)$ \\
\hline $\mathrm{C}(8)$ & $0.0348(14)$ & $0.0283(11)$ & $0.0226(10)$ & $-0.0047(9)$ & $0.0041(11)$ & $0.0003(11)$ \\
\hline $\mathrm{C}(9)$ & $0.0299(13)$ & $0.0357(13)$ & $0.0279(11)$ & $-0.0004(10)$ & $0.0050(11)$ & $-0.0014(11)$ \\
\hline $\mathrm{C}(10)$ & $0.0398(16)$ & $0.0333(13)$ & $0.0342(12)$ & $-0.0020(11)$ & $0.0037(12)$ & $-0.0103(12)$ \\
\hline $\mathrm{C}(11)$ & $0.0518(18)$ & $0.0280(11)$ & $0.0343(12)$ & $-0.0026(11)$ & $0.0034(13)$ & $0.0017(12)$ \\
\hline $\mathrm{C}(12)$ & $0.0371(15)$ & $0.0357(13)$ & $0.0338(13)$ & $-0.0030(11)$ & $-0.0019(12)$ & $0.0060(12)$ \\
\hline$C(13)$ & $0.0336(14)$ & $0.0321(12)$ & $0.0319(12)$ & $-0.0069(10)$ & $0.0009(12)$ & $-0.0023(12)$ \\
\hline$C(14)$ & $0.0453(16)$ & $0.0335(13)$ & $0.0401(14)$ & $-0.0039(11)$ & $0.0089(14)$ & $-0.0118(13)$ \\
\hline $\mathrm{C}(15)$ & $0.070(2)$ & $0.0475(16)$ & $0.0520(17)$ & $0.0032(14)$ & $0.0168(17)$ & $-0.0169(17)$ \\
\hline$C(16)$ & $0.0284(13)$ & $0.0366(13)$ & $0.0380(13)$ & $0.0067(10)$ & $-0.0058(12)$ & $-0.0068(12)$ \\
\hline $\mathrm{C}(17)$ & $0.0310(13)$ & $0.0242(10)$ & $0.0296(12)$ & $0.0004(9)$ & $-0.0041(10)$ & $-0.0020(10)$ \\
\hline $\mathrm{C}(18)$ & $0.0462(16)$ & $0.0325(13)$ & $0.0289(12)$ & $0.0007(10)$ & $0.0039(12)$ & $0.0041(12)$ \\
\hline $\mathrm{C}(19)$ & $0.0484(17)$ & $0.0407(14)$ & $0.0444(14)$ & $0.0042(12)$ & $0.0054(14)$ & $0.0157(14)$ \\
\hline $\mathrm{C}(20)$ & $0.0504(17)$ & $0.0405(14)$ & $0.0405(14)$ & $0.0044(12)$ & $-0.0088(14)$ & $0.0117(15)$ \\
\hline$C(21)$ & $0.0575(18)$ & $0.0379(13)$ & $0.0286(12)$ & $0.0038(11)$ & $0.0010(13)$ & $0.0036(14)$ \\
\hline $\mathrm{C}(22)$ & $0.0379(14)$ & $0.0354(12)$ & $0.0320(12)$ & $0.0004(10)$ & $0.0057(12)$ & $-0.0005(12)$ \\
\hline
\end{tabular}

Table 5: Bond lengths ( $(\AA)$

\begin{tabular}{|c|c|c|c|}
\hline $\mathrm{N}(1)-\mathrm{C}(1)$ & $1.394(3)$ & $\mathrm{C}(8)-\mathrm{C}(9)$ & $1.387(3)$ \\
\hline $\mathrm{N}(1)-\mathrm{C}(3)$ & $1.480(3)$ & $\mathrm{C}(8)-\mathrm{C}(13)$ & $1.392(4)$ \\
\hline $\mathrm{N}(1)-\mathrm{C}(4)$ & $1.389(3)$ & $\mathrm{C}(9)-\mathrm{C}(10)$ & $1.396(4)$ \\
\hline $\mathrm{C}(1)-\mathrm{O}(1)$ & $1.196(3)$ & $\mathrm{C}(10)-\mathrm{C}(11)$ & $1.380(4)$ \\
\hline
\end{tabular}




\begin{tabular}{|c|c|c|c|}
\hline $\mathrm{C}(1)-\mathrm{O}(2)$ & $1.344(3)$ & $\mathrm{C}(11)-\mathrm{C}(12)$ & $1.383(4)$ \\
\hline $\mathrm{O}(2)-\mathrm{C}(2)$ & $1.455(3)$ & $\mathrm{C}(12)-\mathrm{C}(13)$ & $1.394(4)$ \\
\hline $\mathrm{C}(2)-\mathrm{C}(3)$ & $1.520(3)$ & $\mathrm{C}(14)-\mathrm{C}(15)$ & $1.311(4)$ \\
\hline $\mathrm{C}(3)-\mathrm{C}(16)$ & $1.535(3)$ & $\mathrm{C}(16)-\mathrm{C}(17)$ & $1.506(3)$ \\
\hline $\mathrm{C}(4)-\mathrm{O}(3)$ & $1.211(3)$ & $\mathrm{C}(17)-\mathrm{C}(18)$ & $1.390(4)$ \\
\hline $\mathrm{C}(4)-\mathrm{C}(5)$ & $1.529(3)$ & $\mathrm{C}(17)-\mathrm{C}(22)$ & $1.394(3)$ \\
\hline $\mathrm{C}(5)-\mathrm{C}(6)$ & $1.538(3)$ & $\mathrm{C}(18)-\mathrm{C}(19)$ & $1.383(4)$ \\
\hline $\mathrm{C}(5)-\mathrm{C}(7)$ & $1.541(3)$ & $\mathrm{C}(19)-\mathrm{C}(20)$ & $1.390(4)$ \\
\hline $\mathrm{C}(6)-\mathrm{F}(1)$ & $1.410(3)$ & $\mathrm{C}(20)-\mathrm{C}(21)$ & $1.375(4)$ \\
\hline $\mathrm{C}(6)-\mathrm{C}(14)$ & $1.479(4)$ & $\mathrm{C}(21)-\mathrm{C}(22)$ & $1.385(4)$ \\
\hline $\mathrm{C}(7)-\mathrm{C}(8)$ & $1.517(3)$ & & \\
\hline
\end{tabular}

Note $-\mathrm{H}$ atoms have been excluded

Table 6: Bond angles $\left({ }^{\circ}\right)$

\begin{tabular}{|c|l|c|c|}
\hline $\mathrm{C}(1)-\mathrm{N}(1)-\mathrm{C}(3)$ & $110.54(18)$ & $\mathrm{C}(5)-\mathrm{C}(7)-\mathrm{C}(8)$ & $111.97(18)$ \\
\hline $\mathrm{C}(1)-\mathrm{N}(1)-\mathrm{C}(4)$ & $129.8(2)$ & $\mathrm{C}(7)-\mathrm{C}(8)-\mathrm{C}(9)$ & $121.6(2)$ \\
\hline $\mathrm{C}(3)-\mathrm{N}(1)-\mathrm{C}(4)$ & $119.51(19)$ & $\mathrm{C}(7)-\mathrm{C}(8)-\mathrm{C}(13)$ & $119.2(2)$ \\
\hline $\mathrm{N}(1)-\mathrm{C}(1)-\mathrm{O}(1)$ & $128.7(2)$ & $\mathrm{C}(9)-\mathrm{C}(8)-\mathrm{C}(13)$ & $119.1(2)$ \\
\hline $\mathrm{N}(1)-\mathrm{C}(1)-\mathrm{O}(2)$ & $108.77(19)$ & $\mathrm{C}(8)-\mathrm{C}(9)-\mathrm{C}(10)$ & $120.4(2)$ \\
\hline $\mathrm{O}(1)-\mathrm{C}(1)-\mathrm{O}(2)$ & $122.6(2)$ & $\mathrm{C}(9)-\mathrm{C}(10)-\mathrm{C}(11)$ & $120.1(2)$ \\
\hline $\mathrm{C}(1)-\mathrm{O}(2)-\mathrm{C}(2)$ & $110.55(19)$ & $\mathrm{C}(10)-\mathrm{C}(11)-\mathrm{C}(12)$ & $119.9(2)$ \\
\hline $\mathrm{O}(2)-\mathrm{C}(2)-\mathrm{C}(3)$ & $104.72(19)$ & $\mathrm{C}(11)-\mathrm{C}(12)-\mathrm{C}(13)$ & $120.1(2)$ \\
\hline $\mathrm{N}(1)-\mathrm{C}(3)-\mathrm{C}(2)$ & $100.57(18)$ & $\mathrm{C}(8)-\mathrm{C}(13)-\mathrm{C}(12)$ & $120.3(2)$ \\
\hline $\mathrm{N}(1)-\mathrm{C}(3)-\mathrm{C}(16)$ & $111.4(2)$ & $\mathrm{C}(6)-\mathrm{C}(14)-\mathrm{C}(15)$ & $123.0(3)$ \\
\hline $\mathrm{C}(2)-\mathrm{C}(3)-\mathrm{C}(16)$ & $114.8(2)$ & $\mathrm{C}(3)-\mathrm{C}(16)-\mathrm{C}(17)$ & $114.5(2)$ \\
\hline $\mathrm{N}(1)-\mathrm{C}(4)-\mathrm{O}(3)$ & $118.7(2)$ & $\mathrm{C}(16)-\mathrm{C}(17)-\mathrm{C}(18)$ & $120.9(2)$ \\
\hline $\mathrm{N}(1)-\mathrm{C}(4)-\mathrm{C}(5)$ & $120.0(2)$ & $\mathrm{C}(16)-\mathrm{C}(17)-\mathrm{C}(22)$ & $120.8(2)$ \\
\hline $\mathrm{O}(3)-\mathrm{C}(4)-\mathrm{C}(5)$ & $121.24(19)$ & $\mathrm{C}(18)-\mathrm{C}(17)-\mathrm{C}(22)$ & $118.3(2)$ \\
\hline $\mathrm{C}(4)-\mathrm{C}(5)-\mathrm{C}(6)$ & $108.48(19)$ & $\mathrm{C}(17)-\mathrm{C}(18)-\mathrm{C}(19)$ & $121.1(2)$ \\
\hline $\mathrm{C}(4)-\mathrm{C}(5)-\mathrm{C}(7)$ & $109.1(2)$ & $\mathrm{C}(18)-\mathrm{C}(19)-\mathrm{C}(20)$ & $120.0(3)$ \\
\hline $\mathrm{C}(6)-\mathrm{C}(5)-\mathrm{C}(7)$ & $109.79(18)$ & $\mathrm{C}(19)-\mathrm{C}(20)-\mathrm{C}(21)$ & $119.4(3)$ \\
\hline $\mathrm{C}(5)-\mathrm{C}(6)-\mathrm{F}(1)$ & $106.85(18)$ & $\mathrm{C}(20)-\mathrm{C}(21)-\mathrm{C}(22)$ & $120.7(2)$ \\
\hline $\mathrm{C}(5)-\mathrm{C}(6)-\mathrm{C}(14)$ & $113.3(2)$ & $\mathrm{C}(17)-\mathrm{C}(22)-\mathrm{C}(21)$ & $120.5(3)$ \\
\hline $\mathrm{F}(1)-\mathrm{C}(6)-\mathrm{C}(14)$ & $108.2(2)$ & & \\
\hline
\end{tabular}

Note $-\mathrm{H}$ atoms have been excluded 
(2S,3R)-2-benzyl-3-fluoropent-4-enoic acid (9)

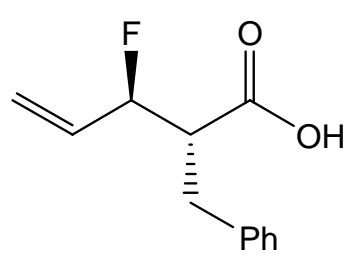

To a solution of compound anti-7 (1.22 mmol, $450 \mathrm{mg})$ in THF $(22 \mathrm{~mL})$ and $\mathrm{H}_{2} \mathrm{O}(7.2$ $\mathrm{mL})$ at $0{ }^{\circ} \mathrm{C}$ was added $\mathrm{H}_{2} \mathrm{O}_{2}(50 \%$ in water, $9.79 \mathrm{mmol}, 0.28 \mathrm{~mL})$ followed by LiOH. $\mathrm{H}_{2} \mathrm{O}(2.45 \mathrm{mmol}, 103 \mathrm{mg})$. The mixture was allowed to warm to room temperature and stirred until no starting material was present $(2 \mathrm{hr})$. The solution is cooled to $0{ }^{\circ} \mathrm{C}$ and quenched with excess saturated $\mathrm{Na}_{2} \mathrm{SO}_{3}$ solution. After evaporation of the THF, the basic solution is extracted with DCM. The solution is then treated with $1 \mathrm{M} \mathrm{HCl}$ until $\sim \mathrm{pH} 2$ and then extracted with ethyl acetate. The crude oil is purified by column chromatography to yield a colourless oil $(95 \%, 240 \mathrm{mg}) ;{ }^{1} \mathrm{H}$ NMR (400 MHz, $\left.\mathrm{CDCl}_{3}\right): \delta=7.14-7.34(\mathrm{~m}, 5 \mathrm{H}), 5.89-6.03(\mathrm{~m}, 2 \mathrm{H}), 5.10(\mathrm{dt}, J=6.8$,

47.0, 1H), 2.85-3.08 (m, 3H); $\left.{ }^{13} \mathrm{C} \mathrm{NMR} \mathrm{(100} \mathrm{MHz,} \mathrm{CDCl}_{3}\right): \delta=177.9,137.6,133.2$ (d, J=19), 128.9, 128.6, 126.8, 120.3 (d, J=12), 93.6 (d, J=175), 52.7 (d, J=22), 33.5 $(\mathrm{d}, J=5) ;{ }^{19} \mathrm{~F}\left\{{ }^{1} \mathrm{H}\right\}$ NMR $\left(376.5 \mathrm{MHz}, \mathrm{CDCl}_{3}\right): \delta=-175.7 ; \mathrm{IR}\left(\mathrm{CDCl}_{3}\right): v 3418,1714$ 1496, 1429, 1224, 988, 699; HMRS required for $\mathrm{C}_{12} \mathrm{H}_{12} \mathrm{O}_{2} \mathrm{~F}$ ([M-H] $\left.]^{-}\right) 207.0821$ found $207.0817 ;[\alpha]_{\mathrm{D}}^{21}=+60.0\left(c, 0.5, \mathrm{CHCl}_{3}\right)$.

\section{(2S, 3S)-2-benzyl-3-fluoropent-4-enoic acid (9)}

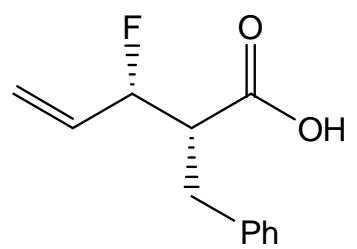

To a solution of compound syn-7 (0.26 mmol, $94 \mathrm{mg})$ in THF $(6 \mathrm{~mL})$ and $\mathrm{H}_{2} \mathrm{O}(2 \mathrm{~mL})$ at $0{ }^{\circ} \mathrm{C}$ was added $\mathrm{H}_{2} \mathrm{O}_{2}(50 \%$ in water, $2.05 \mathrm{mmol}, 0.6 \mathrm{~mL})$ followed by $\mathrm{LiOH} . \mathrm{H}_{2} \mathrm{O}$ 
(0.51 mmol, $22 \mathrm{mg})$. The mixture was allowed to warm to room temperature and stirred until no starting material was present $(2 \mathrm{hr})$. The solution is cooled to $0^{\circ} \mathrm{C}$ and quenched with excess saturated $\mathrm{Na}_{2} \mathrm{SO}_{3}$ solution. After evaporation of the THF, the basic solution is extracted with DCM. The solution is then treated with $1 \mathrm{M} \mathrm{HCl} \mathrm{until}$ $\sim$ pH 2 and then extracted with ethyl acetate. The crude oil was purified by column chromatography to yield a colourless oil $(90 \%, 53 \mathrm{mg}) ;{ }^{1} \mathrm{H}$ NMR (400 MHz, $\left.\mathrm{CDCl}_{3}\right)$ : $\delta=7.19-7.31(\mathrm{~m}, 5 \mathrm{H}), 5.97(\mathrm{dddd}, J=6.3,10.6,14.4,17.1,1 \mathrm{H}), 5.42-5.47(\mathrm{~m}, 1 \mathrm{H})$, 5.36-5.39 (m, 1H), 5.02-5.17 (m, 1H), 3.00-3.06 (m, 3H); ${ }^{13} \mathrm{C}$ NMR $(100 \mathrm{MHz}$ $\left.\mathrm{CDCl}_{3}\right): \delta=176.1,138.0,133.3(\mathrm{~d}, J=19.6), 128.9,128.5,126.7,119.6(\mathrm{~d}, J=11.8)$ $92.6(\mathrm{~d}, J=175.3), 52.3(\mathrm{~d}, J=23.3), 33.27 ;{ }^{19} \mathrm{~F} \mathrm{NMR}\left(376.5 \mathrm{MHz}, \mathrm{CDCl}_{3}\right) \delta-178.3$ to $-178.5(\mathrm{~m}) ; \mathrm{MS} m / z 207.07\left([\mathrm{M}-\mathrm{H}]^{-}\right) ;[\alpha]_{\mathrm{D}}{ }^{21}=+20.0\left(c, 0.15, \mathrm{CHCl}_{3}\right)$.

Synthesis of $(2 S, 3 R)$-2-benzyl-3-fluoropent-4-enoic acid and $(2 S, 3 S)$-2-benzyl-3fluoropent-4-enoic acid (9) from allylsilane (6)
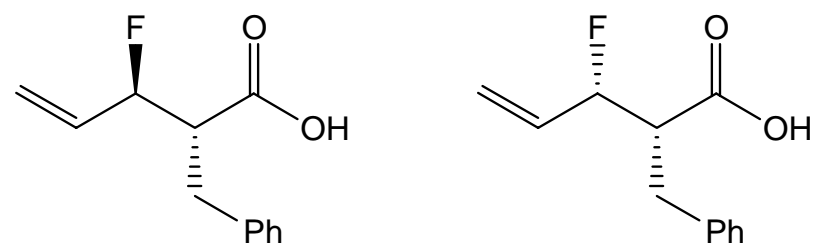

To a solution of 6 in $(0.62 \mathrm{mmol}, 163 \mathrm{mg})$ in $\mathrm{CH}_{3} \mathrm{CN}(3 \mathrm{~mL})$ was added Selectfluor (0.68 mmol, $242 \mathrm{mg}$ ). The reaction was allowed to stir at RT for $48 \mathrm{hrs}$. After column chromatography an inseparable mixture of diastereoisomers was obtained (61\%, 79 $\mathrm{mg})$, anti/syn 1:1. 
(4R)-4-benzyl-3-[(2R)-2-methylbut-3-enoyl]-1,3-oxazolidin-2-one (5)

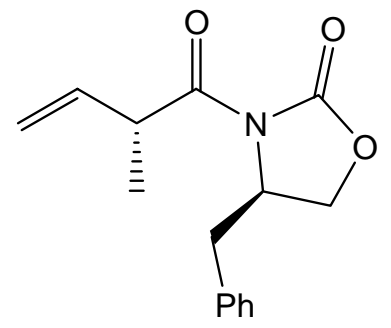

To a stirred solution of diisopropylamine $(4.48 \mathrm{mmol}, 0.63 \mathrm{~mL})$ in THF $(16 \mathrm{~mL})$ at $78{ }^{\circ} \mathrm{C}$ was added n-BuLi ( $\left.1 \mathrm{M}, 4.48 \mathrm{~mL}\right)$. After 10 minutes HMPA (4.48 mmol, 0.78 $\mathrm{mL}$ ) was added and the mixture stirred for 30 minutes at $-78{ }^{\circ} \mathrm{C}$. Compound 3 (4.08 mmol, $1 \mathrm{~g})$ in THF $(8 \mathrm{~mL})$ was added at $-78{ }^{\circ} \mathrm{C}$ followed 15 minutes later by methyl iodide (12.24 mmol, $0.76 \mathrm{~mL})$. After 20 minutes the reaction was allowed to warm to $-10{ }^{\circ} \mathrm{C}$ and stirred at this temperature for 90 minutes. Dilute $\mathrm{HCl}$ was added and the aqueous layer extracted with ether. The combined organic phases were washed with saturated $\mathrm{NaCl}$ solution, dried $\left(\mathrm{MgSO}_{4}\right)$, filtered and the solvent removed in vacuo to yield an orange oil ( $d e$ crude $=42 \%$ ). The two diastereoisomers were separated by column chromatography. The major diastereoisomer was obtained as a colourless oil (476 mg, 45\%). Major diastereoisomer (4R)-4-benzyl-3-[(2R)-2-methylbut-3enoyl]-1,3-oxazolidin-2-one: ${ }^{1} \mathrm{H}$ NMR $\left(400 \mathrm{MHz}, \mathrm{CDCl}_{3}\right): \delta=7.19-7.37(\mathrm{~m}, 5 \mathrm{H})$, 5.99 (ddd, $J=7.6,10.1,17.2), 5.18-5.23(\mathrm{~m}, 1 \mathrm{H}), 5.13-5.16(\mathrm{~m}, 1 \mathrm{H}), 4.63-4.7(\mathrm{~m}$, $1 \mathrm{H}), 4.43-4.5(\mathrm{~m}, 1 \mathrm{H}), 4.16-4.23(\mathrm{~m}, 2 \mathrm{H}), 3.29(\mathrm{dd}, J=3.3,13.4,1 \mathrm{H}), 2.79(\mathrm{dd}, J=$ 9.6, 13.4, 1H), $1.35(\mathrm{~d}, J=7.6,3 \mathrm{H}) ;{ }^{13} \mathrm{C} \mathrm{NMR}\left(100 \mathrm{MHz}, \mathrm{CDCl}_{3}\right): \delta=174.8,152.9$, 136.9, 135.2, 129.4, 128.9, 127.3, 116.6, 66.0, 55.4, 41.7, 37.9, 17.2; IR $\left(\mathrm{CHCl}_{3}\right): v$ 1781, 1217, 756; HMRS required for $\mathrm{C}_{15} \mathrm{H}_{18} \mathrm{NO}_{3}\left([\mathrm{M}+\mathrm{H}]^{+}\right) 260.1287$ found 260.1273; $[\alpha]_{\mathrm{D}}^{21}=-87.3\left(c, 0.5, \mathrm{CHCl}_{3}\right)$. Minor diastereoisomer (4R)-4-benzyl-3[(2S)-2-methylbut-3-enoyl]-1,3-oxazolidin-2-one: ${ }^{1} \mathrm{H}$ NMR $\left(400 \mathrm{MHz}, \mathrm{CDCl}_{3}\right): \delta=$ 7.18-7.37 (m, 5H), $6.0(\mathrm{ddd}, J=7.6,10.4,17.9,1 \mathrm{H}), 5.25-5.3(\mathrm{~m}, 1 \mathrm{H}), 5.18-5.22(\mathrm{~m}$, 
$1 \mathrm{H}), 4.67-4.73(\mathrm{~m}, 1 \mathrm{H}), 4.45-4.54(\mathrm{~m}, 1 \mathrm{H}), 4.15-4.24(\mathrm{~m}, 2 \mathrm{H}), 3.26(\mathrm{dd}, J=3.0,13.1$, $1 \mathrm{H}), 2.76(\mathrm{dd}, J=9.3,13.4,1 \mathrm{H}), 1.32(\mathrm{~d}, J=7.6,3 \mathrm{H}) ;{ }^{13} \mathrm{C} \mathrm{NMR}\left(100 \mathrm{MHz}, \mathrm{CDCl}_{3}\right)$ : $\delta=174.6,152.9,136.9,135.1,129.4,128.9,127.3,116.7,65.9,55.2,41.4,37.7,16.6$ TOF MS CI ${ }^{+} \mathrm{m} / \mathrm{z} 260.121[\mathrm{M}+\mathrm{H}]^{+} ;[\alpha]_{\mathrm{D}}{ }^{21}=-28.9\left(c, 0.65, \mathrm{CHCl}_{3}\right) ; \mathrm{Mp} 72-74{ }^{\circ} \mathrm{C}$. Relative stereochemistry assigned by analogy with compound (7) and literature ref: A. Dobarro, D. Velasco, Tetrahedron 1996, 52, 13525-13530

(4R)-4-benzyl-3-[(2R,3E)-2-methyl-5-(trimethylsilyl)pent-3-enoyl]-1,3-oxazolidin -2-one (2)

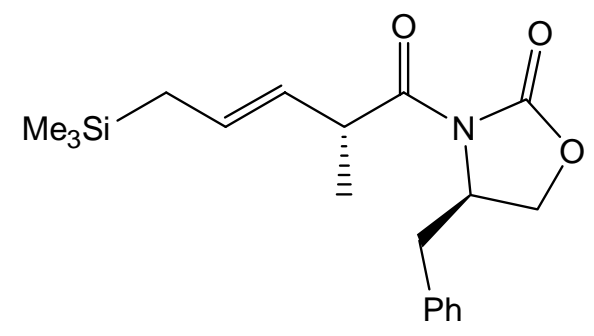

To a solution of 5 (0.65 mmol, $170 \mathrm{mg})$ and allyltrimethyl silane (1.9 mmol, $0.31 \mathrm{~mL})$ in DCM (2 mL) at reflux was added 5 mol\% of Grubbs' second generation catalyst (28mg). After 72 hrs the solvent was removed in vacuo. The crude product was purified by column chromatography to a colourless oil. (83 mg, 77\%) E/Z 3:1. NMR data of the mixture of $E / Z$ isomers. ${ }^{1} \mathrm{H}$ NMR $\left(400 \mathrm{MHz}, \mathrm{CDCl}_{3}\right): \delta=7.21-7.36(\mathrm{~m}$, $5 \mathrm{H}), 5.64(\mathrm{dt}, J=8.1,15.4,1 \mathrm{H}), 5.41-5.58,\left(\mathrm{~m}, 2 \mathrm{H}_{\mathrm{Z}}\right) 5.32-5.39(\mathrm{~m}, 1 \mathrm{H}), 4.68-4.78(\mathrm{~m}$ $\left.1 \mathrm{H}_{\mathrm{Z}}\right) 4.59-4.68(\mathrm{~m}, 1 \mathrm{H}), 4.38-4.48(\mathrm{~m}, 1 \mathrm{H}), 4.14-4.19(\mathrm{~m}, 2 \mathrm{H}), 3.30(\mathrm{dd}, J=3.28$, $13.14,1 \mathrm{H}), 2.78(\mathrm{dd}, J=9.6,13.14,1 \mathrm{H}), 1.48-1.63\left(\mathrm{~m}, 2 \mathrm{H}_{Z}\right), 1.46(\mathrm{~d}, J=8.1,2 \mathrm{H})$, $1.31\left(\mathrm{~d}, J=6.8,3 \mathrm{H}_{\mathrm{Z}}\right), 1.30(\mathrm{~d}, J=6.8,3 \mathrm{H}), 0.0\left(\mathrm{~s}, 9 \mathrm{H}_{\mathrm{Z}}\right),-0.02(\mathrm{~s}, 9 \mathrm{H}) ;{ }^{13} \mathrm{C}$ NMR $(100$ $\left.\mathrm{MHz}, \mathrm{CDCl}_{3}\right): \delta=175.5,152.3,135.4_{\text {Zor } E}, 135.3_{\text {Zor E }}, 129.7_{\text {Zor E }}, 129.5_{\text {Zor E }}, 128.9_{\text {Zor }}$ ${ }_{E}, 128.1_{Z}, 127.3,126.9,126.1_{Z}, 66.03,66.0_{Z}, 55.6,55.5_{Z}, 40.9,37.9,37.8_{Z}, 35.8_{Z}, 22.9$ $19.0_{Z}, 17.9,-1.8_{Z},-1.9 ; \quad \mathrm{IR}\left(\mathrm{CHCl}_{3}\right): v 2955,1783,1699,1454,1381,853 ; \mathrm{HMRS}$ 
required for $\mathrm{C}_{19} \mathrm{H}_{27} \mathrm{NO}_{3} \mathrm{Si}\left([\mathrm{M}+\mathrm{H}]^{+}\right) 346.1838$ found $346.1847 ;[\alpha]_{\mathrm{D}}^{23}=-131.6(c, 2$, $\left.\mathrm{CHCl}_{3}\right)$.

(4R)-4-benzyl-3-[(2S),(3R)-fluoro-2-methylpent-4-enoyl]-1,3-oxazolidin-2-one and (4R)-4-benzyl-3-[(2S),(3S)-fluoro-2-methylpent-4-enoyl]-1,3-oxazolidin-2-one (8)
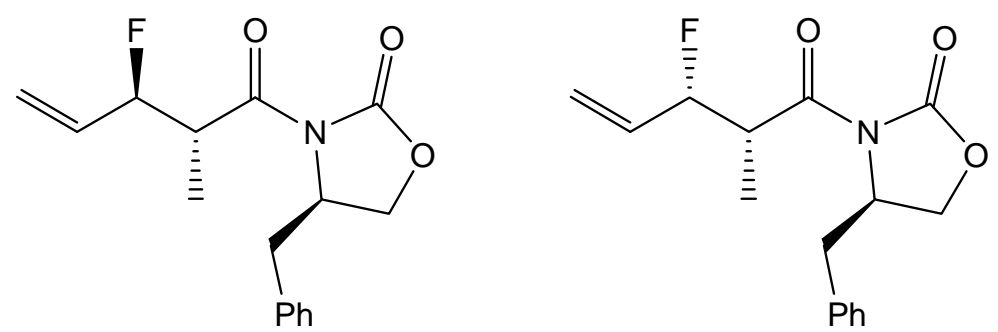

To a solution of 2 ( $0.24 \mathrm{mmol}, 84 \mathrm{mg})$ in $\mathrm{CH}_{3} \mathrm{CN}(2 \mathrm{~mL})$ was added Selectfluor $(0.26$ mmol, $93 \mathrm{mg}$ ). The reaction was allowed to stir at RT for $48 \mathrm{hr}$. The solvent was removed in vacuo. The crude product was purified by column chromatography to yield a mixture of diastereoisomers anti/syn 1:1 (58 mg, 82\%). Anti diastereoisomer ${ }^{1} \mathrm{H}$ NMR (400 MHz, $\left.\mathrm{CDCl}_{3}\right): \delta=7.16-7.42(\mathrm{~m}, 5 \mathrm{H}), 5.88-6.02(\mathrm{~m}, 1 \mathrm{H}), 5.37-5.46(\mathrm{~m}$, $1 \mathrm{H}), 5.29-5.34(\mathrm{~m}, 1 \mathrm{H}), 5.15(\mathrm{dt}, J=5.8,47.0,1 \mathrm{H}), 4.69(\mathrm{~m}, 1 \mathrm{H}), 4.2(\mathrm{~m}, 2 \mathrm{H}), 4.1(\mathrm{~m}$, $1 \mathrm{H}), 3.29(\mathrm{dd}, J=3.3,13.4,1 \mathrm{H}), 2.79(\mathrm{dd}, J=9.6,13.4,1 \mathrm{H}), 1.33(\mathrm{~d}, J=7.1,3 \mathrm{H}) ;{ }^{13} \mathrm{C}$ NMR (125 MHz, $\left.\mathrm{CDCl}_{3}\right): \delta=173.2,153.1,135.0,133.9$ (d, J=20), 129.4, 128.9, 127.4, 118.6 (d, $J=12), 93.1$ (d, $J=174), 66.2,55.4,50.9,42.6$ (d, $J=24), 37.7,11.9$ (d, $J=6) ;{ }^{19} \mathrm{~F} \quad\left\{{ }^{1} \mathrm{H}\right\}$ NMR $\left(376.5 \mathrm{MHz}, \mathrm{CDCl}_{3}\right): \delta=-188.9 ;$ IR $\left(\mathrm{CHCl}_{3}\right): v 2950,1779$, 1700; HMRS required for $\mathrm{C}_{16} \mathrm{H}_{19} \mathrm{NO}_{3} \mathrm{~F}\left([\mathrm{M}+\mathrm{H}]^{+}\right) 292.1349$ found 292.1360 ; $[\alpha]_{\mathrm{D}}^{23}=-$ $38.4\left(c, 0.25, \mathrm{CHCl}_{3}\right)$. Syn diastereoisomer ${ }^{1} \mathrm{H} \mathrm{NMR}\left(400 \mathrm{MHz}, \mathrm{CDCl}_{3}\right): \delta=7.18-7.4$ (m, 5H), 5.8-6.0 (m, 1H), 5.39-5.51 (m, 2H), $5.14(\mathrm{dt}, J=7.8,47.5,1 \mathrm{H}), 4.74(\mathrm{~m}, 1 \mathrm{H})$, $4.23(\mathrm{~m}, 2 \mathrm{H}), 4.12(\mathrm{~m}, 1 \mathrm{H}), 3.28(\mathrm{dd}, J=3.3,13.4,1 \mathrm{H}), 2.82(\mathrm{dd}, J=9.6,13.4,1 \mathrm{H})$ $1.18(\mathrm{~d}, J=7.1,3 \mathrm{H}) ;{ }^{13} \mathrm{C} \mathrm{NMR}\left(125 \mathrm{MHz}, \mathrm{CDCl}_{3}\right): \delta=173.9,153.1,135.1,133.5(\mathrm{~d}$, 
$J=24), 129.4,128.9,127.4,120.8$ (d, $J=15), 94.8$ (d, $J=211), 66.3,55.4,42.1$ (d, $J=29), 37.8,13.5(\mathrm{~d}, J=9) ;{ }^{19} \mathrm{~F}\{1 \mathrm{H}\}$ NMR $\left(376.5 \mathrm{MHz}, \mathrm{CDCl}_{3}\right): \delta=-171.9 ; \mathrm{HMRS}$ required for $\mathrm{C}_{16} \mathrm{H}_{19} \mathrm{NO}_{3} \mathrm{~F}\left([\mathrm{M}+\mathrm{H}]^{+}\right) 292.1349$ found $292.1342 ;[\alpha]_{\mathrm{D}}^{23}=-45.7(c, 1$, $\left.\mathrm{CHCl}_{3}\right) ; \mathrm{Mp} 68-71{ }^{\circ} \mathrm{C}$. Relative stereochemistry of products assigned by analogy with the benzyl substituted allylic fluorides based on the ${ }^{19} \mathrm{~F}\left\{{ }^{1} \mathrm{H}\right\}$ NMR chemical shifts.

\section{(2S,3S)-2-benzyl-3-fluoropent-4-en-1-ol (10)}

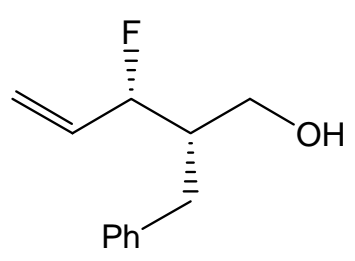

To a stirred suspension of $\mathrm{LiAlH}_{4}(0.25 \mathrm{mmol}, 10 \mathrm{mg})$ in THF $(1 \mathrm{~mL})$ at RT was added syn- $9(0.25 \mathrm{mmol}, 52 \mathrm{mg})$ in THF $(1 \mathrm{~mL})$ dropwise. The reaction was allowed to stir for $14 \mathrm{hr}$ at RT. After cooling to $0^{\circ} \mathrm{C}$, water was added dropwise, followed by $5 \% \mathrm{NaOH}$. After stirring for 30min the mixture was filtered through celite. The solution was then extracted with ethyl acetate. The combined organic extracts were washed then dried over $\mathrm{MgSO}_{4}$ to yield a colourless oil (62\%, $\left.30 \mathrm{mg}\right) ;{ }^{1} \mathrm{H}$ NMR (400 $\left.\mathrm{MHz}, \mathrm{CDCl}_{3}\right): \delta=7.18-7.34(\mathrm{~m}, 5 \mathrm{H}), 5.93-6.07(\mathrm{~m}, 1 \mathrm{H}), 5.34-5.48(\mathrm{~m}, 2 \mathrm{H}), 5.14(\mathrm{dt}$, $J=5.3,47,1 \mathrm{H}), 3.61-3.67(\mathrm{~m}, 2 \mathrm{H}), 2.88(\mathrm{dd}, J=5.1,13.9,1 \mathrm{H}), 2.64(\mathrm{dd}, J=9.6,13.9$ 1H), 2.06-2.21 (m, 1H); ${ }^{13} \mathrm{C}$ NMR (125 MHz, $\left.\mathrm{CDCl}_{3}\right): \delta=139.7,134.7(\mathrm{~d}, J=19)$, 129.1, 128.5, 126.2, 118.0 (d, $J=13), 93.7$ (d, $J=170), 61.3$ (d, $J=3), 47.1$ (d, $J=20)$,

$31.8(\mathrm{~d}, J=6) ;{ }^{19} \mathrm{~F}\left\{{ }^{1} \mathrm{H}\right\}$ NMR $\left(376.5 \mathrm{MHz}, \mathrm{CDCl}_{3}\right): \delta=-188.9 ; \mathrm{IR}\left(\mathrm{CHCl}_{3}\right): v 3383$, 2930; HMRS required for $\mathrm{C}_{12} \mathrm{H}_{19} \mathrm{NOF}\left(\left[\mathrm{M}+\mathrm{NH}_{4}\right]^{+}\right)$212.1451 found 212.1448. 